\title{
Evaluation of optimal sensor placement algorithms for the Structural Health Monitoring of architectural heritage. Application to the Monastery of San Jerónimo de Buenavista (Seville, Spain).
}

\author{
Pablo Pachón ${ }^{\mathrm{a}, *}$, María Infantes ${ }^{\mathrm{b}}$, Margarita Cámara ${ }^{\mathrm{a}}$, Víctor Compán ${ }^{\mathrm{a}}$, Enrique García-Macías ${ }^{\mathrm{e}}$, \\ Michael I. Friswell ${ }^{\text {, Rafael Castro-Triguero }}{ }^{\mathrm{b}}$ \\ ${ }^{a}$ Department of Continuum Mechanics, Universidad de Sevilla, Avenida Reina Mercedes, 41012 Seville, Spain \\ ${ }^{b}$ Department of Mechanics, Universidad de Córdoba, Campus de Rabanales, 14071 Cordoba, Spain \\ ${ }^{c}$ Department of Continuum Mechanics and Structural Analysis, Universidad de Sevilla, Camino de los \\ Descubrimientos s/n, 41092 Seville, Spain \\ ${ }^{d}$ Zienkiewicz Centre for Computational Engineering, College of Engineering, Swansea University, Bay Campus, \\ Fabian Way, Crymlyn Burrows, Swansea, SA1 8EN, United Kingdom \\ ${ }^{e}$ Department of Civil and Environmental Engineering, University of Perugia, Via G Duranti 93, Perugia 06125, \\ Italy
}

\begin{abstract}
In recent years, Structural Health Monitoring (SHM) based on Operational Modal Analysis (OMA) and damage detection tools has become a popular non-destructive solution to assess the real-time integrity of any kind of structure. This technique is especially well-suited for the condition-based conservation of historical structures, where minimal invasiveness must be ensured owing to their high cultural and architectural value. Optimal Sensor Placement (OSP) techniques represent a valuable tool for efficiently designing the sensor layout in a SHM system in order to achieve an effective modal identification with a reduced number of sensors and, consequently, an improved cost efficiency. In this light, this paper proposes a design methodology of sensor networks based on OSP techniques suitable for historical structures. To do so, a preliminary extensive OMA campaign is conducted in order to construct a reliable finite element (FE) model by fitting the identified modal properties. Afterwards, an optimal sensor arrangement with a limited number of sensors is obtained by applying different model-based OSP techniques. In order to improve the robustness of the solution, material uncertainties are included in the model and the optimal sensor placement is conducted within a statistical framework. This methodology is presented and evaluated with a case study of a Spanish secular building: the Monastery of San Jerónimo de Buenavista in Seville (Spain). In particular, this paper presents the results of the preliminary ambient vibration test and the modal identification of the monastery, the updating process of the FE model, as well as a critical review of the different OSP techniques within a framework of material parameter uncertainty. The presented analysis demonstrate that OSP techniques based on the rank optimization of the kinetic energy matrix of the structure yield robust sensor layout.
\end{abstract}

Keywords:

Historical building, Uncertainty analysis, Operational modal analysis, Non-destructive test, Ambient vibration test 


\section{Introduction}

Architectural heritage in Europe consists mainly of masonry structures, such as historical bridges, castles, palaces and religious buildings. These structures are part of the national history and hold an important cultural value, as confirmed by the creation of the World Heritage List published by UNESCO. In the Spanish context, many of these still standing heritage buildings were pillaged and burned over the years, whereby their structural integrity is not always assured. The conservation and safeguarding of historical structures against material degradation and natural/human hazards represent a great concern for communities and institutions such as the International Council of Monuments and Sites (ICOMOS), which in 2003 drafted the principles for the analysis, conservation and structural restoration of architectural heritage [1].

The assessment of the health condition of historical buildings cannot usually be carried out by means of destructive tests due to the undeniable value of the historical structures. In this context, non-destructive and non-invasive methods become a fundamental technique for this type of structures. Non Destructive Tests (NDTs) such as thermography, endoscopic tests and sonic tests can be used as complementary tools to accurately determine the characteristics of masonry elements in order to validate the structural assessment $[2,3]$. However, foremost among the different NDTs are OMA and model updating. These techniques allow the assessment of the structural condition through the identification and analysis of the modal properties. System identification is a research topic with decades of history, particularly in aerospace and automotive industries, where Experimental Modal Analysis (EMA) is widely used for the dynamic characterization of structural elements from input-output vibration experimental data. The implementation of this philosophy into large structures, where ambient vibration tests are easier to carry out than forced vibration tests, gave rise to output-only vibration-based system identification methods, also referred to as OMA techniques. The main objective of OMA is to identify the modal parameters (natural frequencies, vibration modes and damping ratios) through the data processing of ambient vibration records without disturbing the normal operation of the structure under study. Some basic references regarding different OMA methods and applications are [4-6]. From early approaches to now, the measurement equipment has evolved and many different algorithms have been proposed in the literature including Frequency Domain Decomposition (FDD) and Enhanced Frequency Domain Decomposition (EFDD), Fast Bayesian FFT method, Poly-Reference Least Squares Complex Frequency method (p-LSCF), Covariance-Driven Stochastic System Identification (COV-SSI), Data-Driven Stochastic System Identification (DATA-SSI), Blind Source Separation, etc. Nowadays, reliable and even automated methods are available [7], making OMA a well-established and mature technique for the structural assessment of heritage structures. The consideration of this methodology for the characterization of masonry structures is relatively recent. The $\mathrm{PhD}$ thesis of Ramos at the University of Minho [8] can be cited as one of the first approaches. OMA has been successfully used in [9] to identify the modal properties of a building of the Greek heritage and also in the dynamic identification of the bell tower of Trani's Cathedral [10]. Modal updating techniques seek to minimize the mismatch between the experimentally identified modal features and the estimates of a numerical model, usually based on the Finite Element Method (FEM), through the fitting of certain modal parameters [11-14].

In the last decade, long-term vibration-based SHM is becoming increasingly popular among

\footnotetext{
${ }^{*}$ Corresponding author.

Email address: ppachon@us.es (Pablo Pachón)
} 
researchers. This non-invasive technique is mainly based on OMA techniques and makes it possible to identify structural damage over time. In reference [15], SHM technology and automated OMA techniques are specially applied to structures in seismically prone areas to evaluate the dynamic behaviour in operational conditions and the earthquake effects. Currently, there are few SHM systems installed in historical masonry structures that are faithful to the steps of the paradigm defined by Farrar and Worden [16]. Initially, most monitoring systems were implemented in bell towers, since in this type of structures the dynamic properties can be easily identified with moderate execution costs [17-19]. In recent years, these techniques have been extended to other typologies of greater geometric complexity which involve structural models with larger computational demands $[20,21]$. In general, SHM systems aim to control the structural behaviour of these buildings to identify damage and assist decision-making for condition-based conservation management [22-24].

To facilitate the implementation of SHM systems in historical structures, OSP methods constitute a useful tool. The main goal of this technique is to efficiently design the sensor layout to achieve a correct identification of the vibrational properties with a reduced number of measurement points. The reduction of the number of sensors is a very important issue in historical structures because it implies less invasive monitoring, and minimal impact on the cultural and artistic value of the building. Furthermore, it reduces the implementation costs and the posterior data processing. The efficient design of the sensor layout is particularly relevant in geometrically complex structures for which mode shapes are also complex and involve several global and local modes within the frequency range of interest [25]. In these cases, a rational approach for the design of SHM systems can certainly take advantage of OSP techniques in order to achieve an effective modal identification. Optimal sensor placement methods have been widely employed in civil engineering structures such as bridges, whereas the number of applications in historical structures is scarce. Only a few experiences can be found in the literature [26-28]. In addition, most works on the application of OSP techniques are based upon simple benchmark case studies and/or consider deterministic definitions of the material properties of the monitored structure.

In this work, four different FEM-based OSP methodologies are considered, including two approaches based on the effective independence of the target mode shapes (EFI and EFIwm), and two others based on the concept of energy matrix rank optimization (KEMRO and SEMRO). The main objective is to evaluate the performance of the different OSP methods, especially when taking into account the particular aspects involved in the numerical modelling and monitoring of historical structures. The analysis of the OSP methods is performed by considering field data and including material parameter uncertainties in the modelling. This research is carried out through a case study of the Monastery of San Jerónimo de Buenavista (Fig. 1) in Seville, Spain. First, an extensive ambient vibration test was conducted for a preliminary identification of the modal features of the structure. Afterwards, a detailed three-dimensional finite element model of the monastery is calibrated to fit the previously identified modal properties and, finally, the updated model is used to assist different OSP techniques. The effect of material parameter uncertainties is evaluated through Monte Carlo Simulations.

The main novelty of this work is the evaluation of the potentials of different OSP techniques for the design of optimized sensor layout for the condition-based maintenance of historical buildings. In the first place, the use of field data from a preliminary ambient vibration test with many sensors offers a rigorous framework for the comparison of different OSP techniques and represents an advanced technique for devising cost-efficient long-term monitoring systems. Secondly, the incorporation of material parameter uncertainties in the three-dimensional modelling of masonry 
structures, and the comparison of different OSP techniques through Monte Carlo Simulations also represents a novel contribution in the field. The paper is organized as follows. Section 2 presents the case study of the Monastery of San Jerónimo de Buenavista, including a detailed description of its historical background and current condition. Section 3 shows the experimental structural assessment works (ambient vibration tests, OMA) and the model updating process. The optimal number of sensors for a long-term SHM system and the analysis of the four different OSP methods are addressed in Section 4, and the analysis of their robustness considering material uncertainties is presented in Section 5. Finally, the most relevant conclusions of this study are highlighted in Section 6.

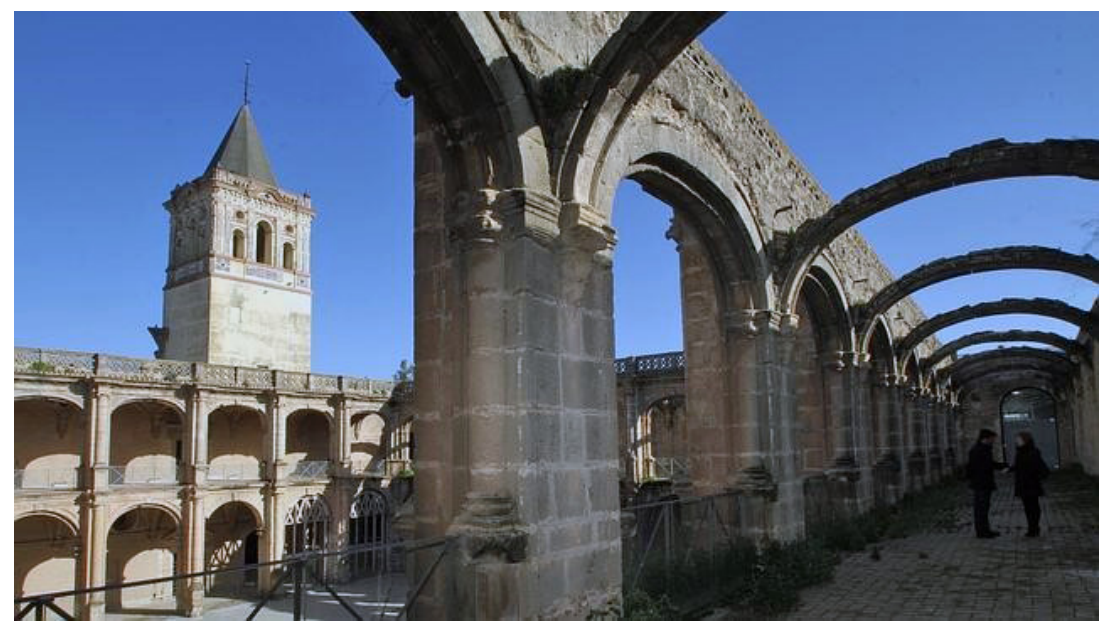

Fig. 1: View of the current state of the Monastery of San Jerónimo de Buenavista.

\section{Monastery of San Jerónimo de Buenavista}

This study focuses on the west gallery of the main cloister of the Monastery of San Jerónimo de Buenavista, a historical building located in the city of Seville, Spain. The beginning of its construction dates back to the early fifteenth century. Originally, the Monastery of San Jerónimo consisted of an aggregate of five main buildings: the church, the eastern cloister, the main cloister, the tower and the printing press (Fig. 2a). However, only part of the main cloister and the tower of the church are still preserved today (Fig. 2b). The eastern cloister, the printing press and a large part of the church disappeared as a result of the numerous vicissitudes that the monastery has suffered over the course of centuries [29]. 


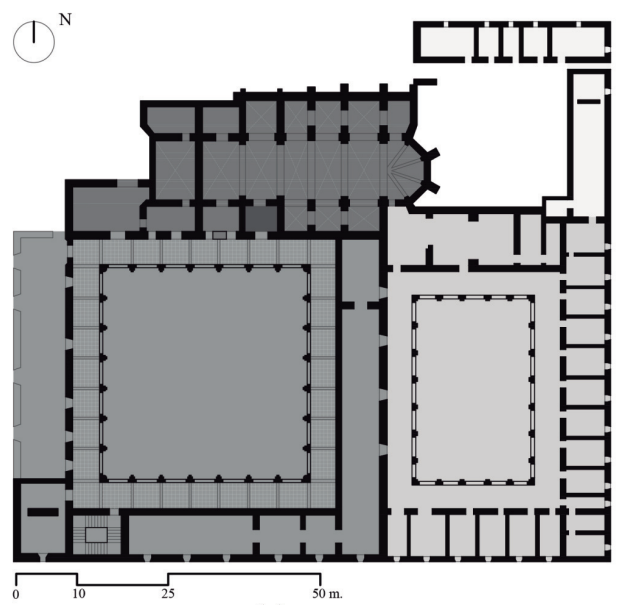

(a)

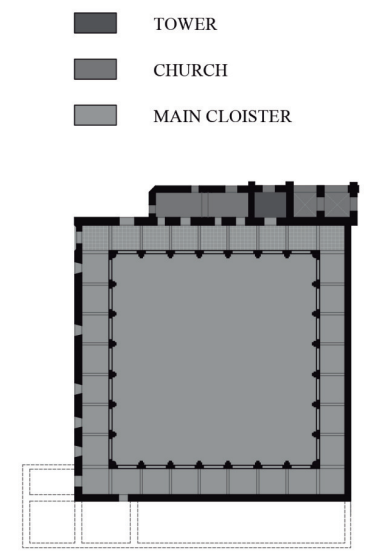

(b)

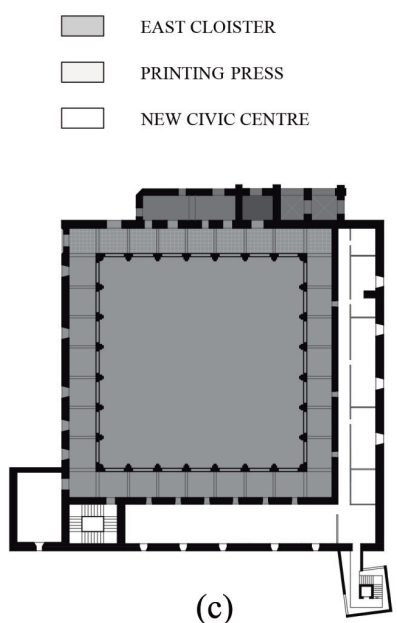

(c)

Fig. 2: Historical development of the Monastery of San Jerónimo: (a) Original configuration, 1650; (b) historical remains, 2000; and (c) current architectural configuration of the complex, 2018.

The remains of the main cloister constitute a Renaissance-style construction consisting of four galleries, which are made up of seven pillars on each side, attached columns and semicircular arches on the ground floor and bells on the first floor. These galleries are delimited by brick masonry walls and covered by ribbed stone vaults. Currently, in the west gallery, the entire ground floor is conserved while only the vertical structure and the arches are preserved on the upper floor (Fig. $3)$.
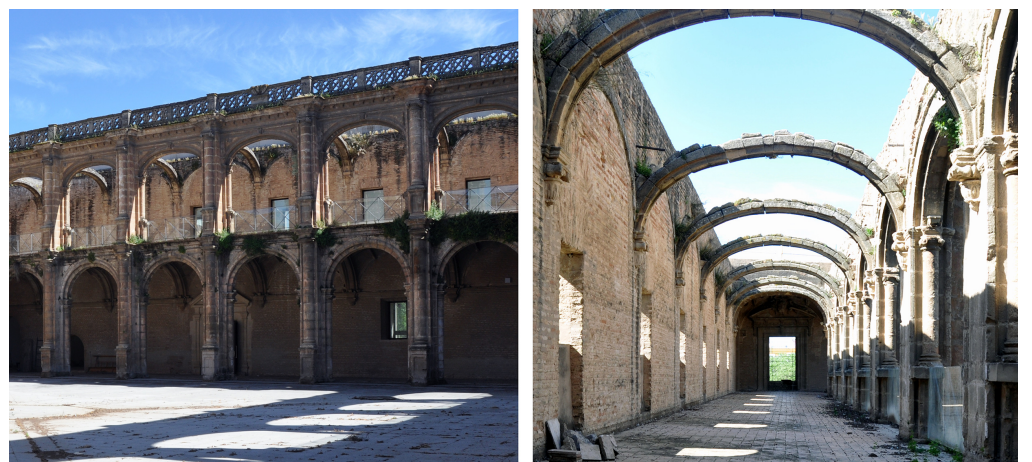

Fig. 3: Views of the west gallery of the Monastery of San Jerónimo.

In 1964, the monastery was declared a heritage site due to the architectural importance of the whole building. Since then, it has been subjected to several restoration operations [29]. The last one was the execution of a rehabilitation and adaptation project for a civic centre in 2013 (Fig. 2c). The project basically consisted of the reconstruction of the southern and eastern galleries annexed to the main cloister [30]. From a structural point of view, a mixed system of load-bearing walls and pillars supporting large-edged beams was used to construct the new building (Fig. 4). 

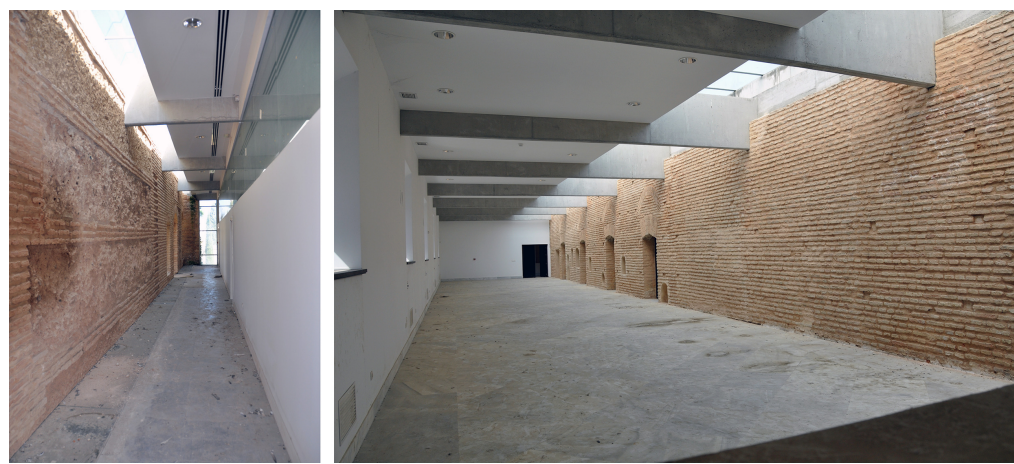

Fig. 4: Views of the civic centre annexed to the Monastery of San Jerónimo.

As a result of the new protection policy for the Monastery of San Jerónimo, several damages had already been detected before the construction of the new civic centre. These are related to the following aspects: cracking of structural elements, cracking in auxiliary elements, excessive deformations, failures in foundations, concentration of humidity, corrosion of metallic elements, disintegration, cracking, factory swelling and attacks, etc. (Fig. 5). In January 2015, and due to the fact that some of the damages listed above were aggravated during the works, the Town Planning Department of Seville City Council commissioned a research project for the structural analysis of the cloister.
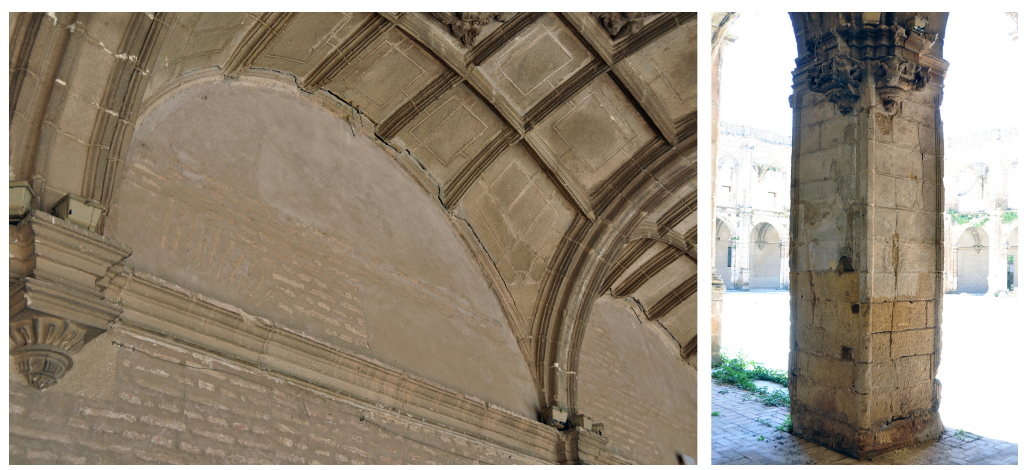

Fig. 5: Detected damage in the main cloister of the Monastery of San Jerónimo.

The research project indicated above follows the approach ratified by the ICOMOS 14th General Assembly in Victoria Falls, Zimbabwe, in 2003 [1]. The main objective was to conduct a diagnosis of the building to justify different conservation proposals to ensure the integrity of all its elements. A multidisciplinary work team composed of architects, engineers, archaeologists and historians carried out the research project. An extensive historical study of the different interventions in the building was finally presented, as well as a report about its structural health condition. To elaborate this report, complex numerical analysis and several moderately destructive and non-destructive tests were conducted, including ambient vibration tests, archaeological tastings, tomography and sonic wave tests and georadar tests on the columns and ground. This project allowed us to obtain a vast amount of experimental and numerical information from the west gallery of the cloister. The data obtained from these previous works were fundamental for the development of the present study, since it was possible to reduce the high level of uncertainty that usually surrounds the structural characterisation of this type of buildings. 


\section{FE modelling and analysis}

The reparation works in the historical building allowed us to perform several extensive experimental campaigns in the monastery between September and November 2013. Ambient vibration tests were performed in the west gallery with the aim of identifying the natural frequencies, mode shapes and damping ratios of the system. In order to ensure an efficient identification of the modal properties of the case study, a preliminary finite element model was previously developed with the aim of properly designing the test set-up. This numerical model was later updated based on the results obtained from the OMA of the ambient vibration tests. This model updating process is described in sections below.

\subsection{Preliminary FE model}

Based on a complex geometric survey, a sophisticated three-dimensional FE model of the whole monastery was developed in order to conduct a subsequent structural analysis that is not a subject of this work. Initially, the modal behaviour of the entire structure was analysed. The results of the modal analysis of the monastery is depicted in Fig. 6.

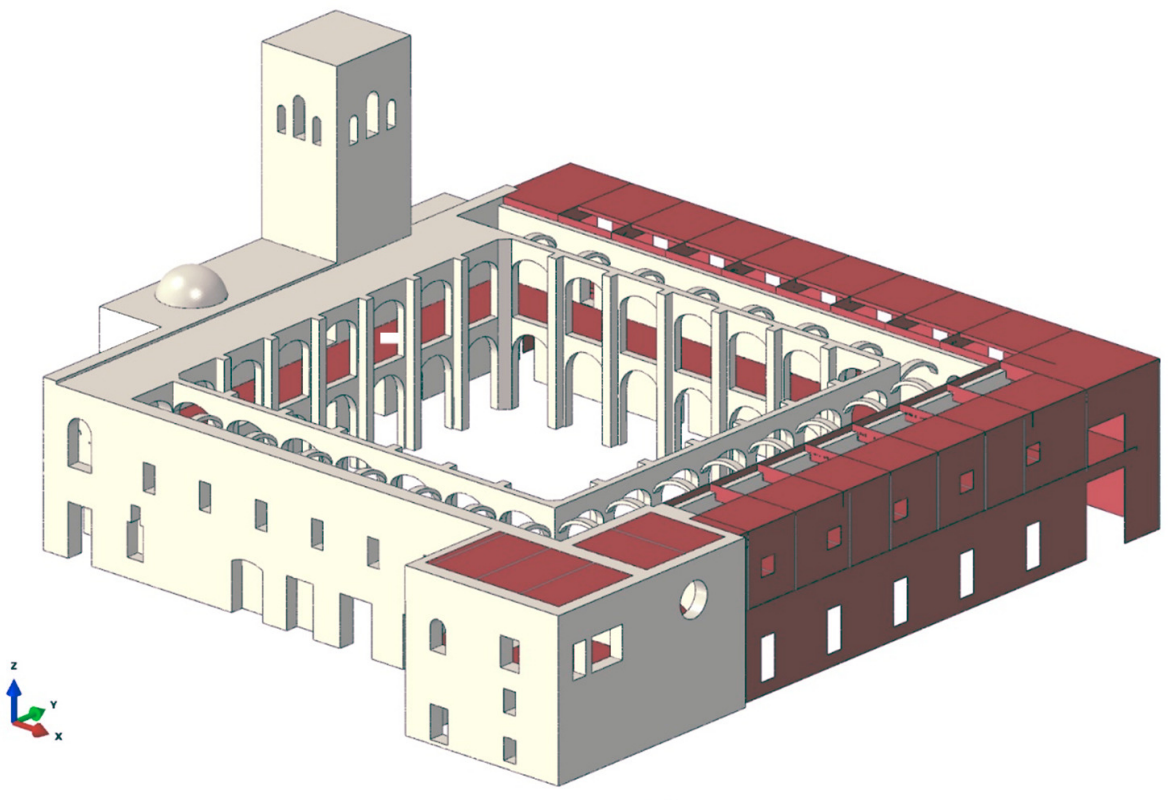

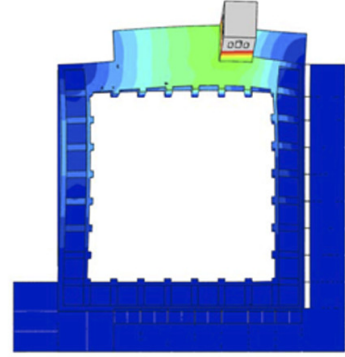

$1^{\text {st }}$ G. Mode $(2.02 \mathrm{~Hz})$

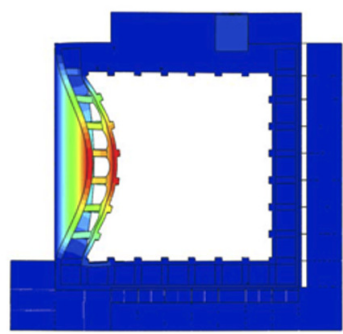

$2^{\text {nd }} \mathrm{G}$. Mode $(2.33 \mathrm{~Hz})$

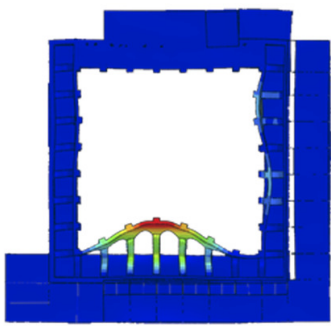

$5^{\text {st }} \mathrm{G}$. Mode $(4.07 \mathrm{~Hz})$

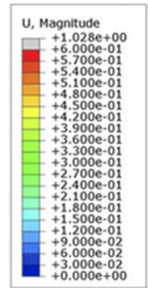

$3^{\text {rd }}$ G. Mode $(3.13 \mathrm{~Hz})$

$4^{\text {th }} \mathrm{G}$. Mode $(3.60 \mathrm{~Hz})$

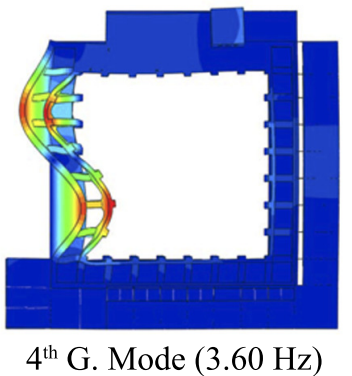

Fig. 6: Three-dimensional FE model of the monastery and numerical identification of the first five global modes.

In the light of this analysis, the dynamic independence of each gallery was observed, since each one presents its own uncoupled vibration modes. For instance, the second and fourth global modes 
correspond to decoupled modes of the west gallery. Regarding that the goal of the study is the evaluation of the different OSP techniques, it was decided to perform the analysis considering only the west gallery due to the large size of the complete model. This last consideration makes it possible to reduce the computational cost of the study without losing generality in the evaluation of the different OSP techniques for their application in masonry structures. A numerical sub-model of this gallery (Fig. 7) was generated with ANSYS [31]. The influence of the other parts of the structure were considered through boundary conditions.
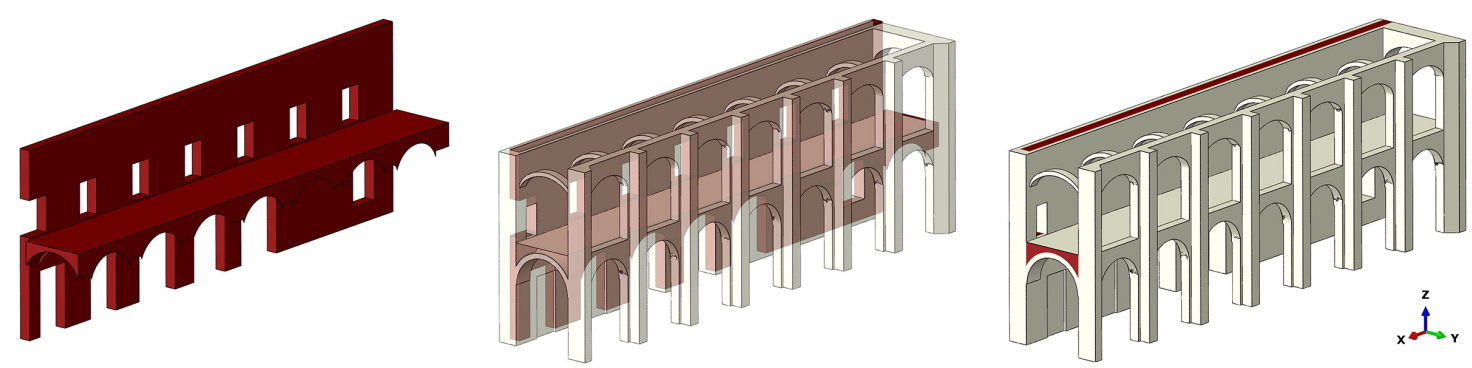

Fig. 7: Three-dimensional FE model of the west gallery.

With regard to the numerical definition of the preliminary FE model, the whole gallery was modelled with 4-node solid elements, with the exception of a 5-centimetre concrete compression layer located above the vaults which was modelled with 3 -node triangular shell elements. In this way, the model has a total of 1462311 elements (1454047 solid and 8264 shell elements), 275475 nodes and 826425 degrees of freedom. The boundary conditions are defined as constrained displacements and free rotations in the foundation, whilst the lateral boundary conditions are extracted from the general model. Table 1 summarizes the material properties used in the modelling. Note that the repair works allowed us to reliably test some elements, such as the material properties of the fillings. Dynamic tests were also carried out on the stone and brick masonries to identify the Young's moduli and densities of these elements. However, it was not possible to determine these parameters with certainty due to the great variability of the obtained results. Thus, these four parameters will be subsequently selected as the variables to be updated. 
Table 1: Material properties used in the preliminary FE model.

\begin{tabular}{lcc}
\hline Property & Unit & Value \\
\hline Young's modulus of brick masonry (wall) & $\mathrm{MPa}$ & 1300 \\
Poisson's ratio of brick masonry & - & 0.2 \\
Density of brick masonry & $\mathrm{kg} / \mathrm{m}^{3}$ & 1700 \\
Young's modulus of stone masonry (vaults and columns) & $\mathrm{MPa}$ & 1800 \\
Poisson's ratio of stone masonry & - & 0.2 \\
Density of stone masonry & $\mathrm{kg} / \mathrm{m}^{3}$ & 2000 \\
Young's modulus of concrete slab & $\mathrm{MPa}$ & 23000 \\
Poisson's ratio of concrete slab & - & 0.2 \\
Density of concrete slab & $\mathrm{kg} / \mathrm{m}^{3}$ & 2500 \\
Young's modulus of filling (wall) & $\mathrm{MPa}$ & 500 \\
Poisson's ratio of filling (wall) & - & 0.2 \\
Density of filling (wall) & $\mathrm{kg} / \mathrm{m}^{3}$ & 1500 \\
Young's modulus of filling (vaults) & $\mathrm{MPa}$ & 350 \\
Poisson's ratio of filling (vaults) & - & 0.2 \\
Density of filling (vaults) & $\mathrm{kg} / \mathrm{m}^{3}$ & 900 \\
\hline
\end{tabular}

As noted above, a modal analysis of this preliminary FE model was performed to identify which points experience larger modal displacements in as many vibration modes as possible. The first five mode shapes are shown in Fig. 8. It is observed that the complex geometric configuration of the arches on the first floor makes the dynamic features of the structure quite complex.
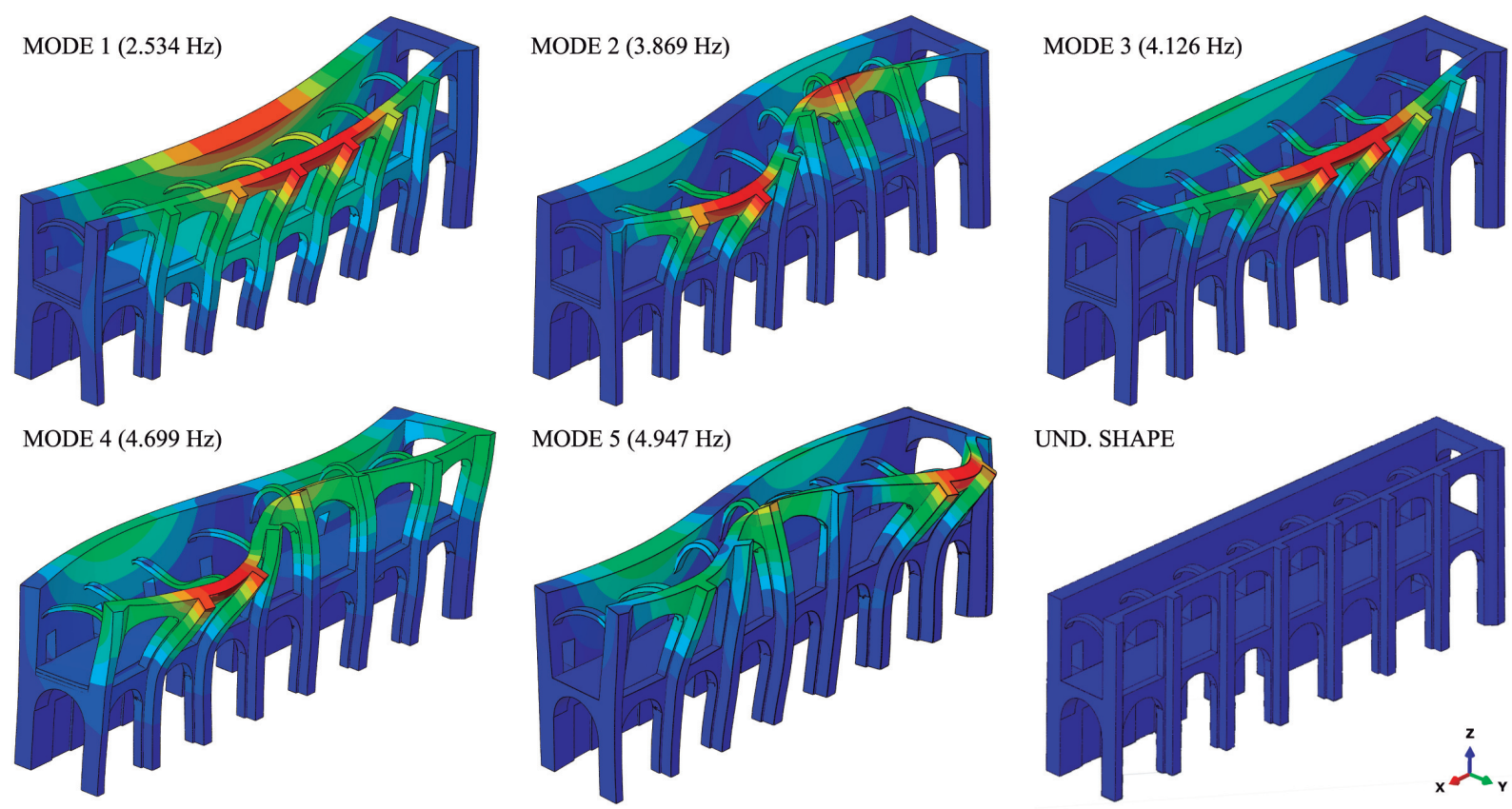

Fig. 8: First five numerical vibration modes and undeformed configuration of the FE model. 


\subsection{Ambient vibration tests}

An extensive ambient vibration campaign with a large number of sensors was performed on the west gallery on November 22th, 2013. Following the results obtained from the preliminary FE model, Fig. 9 shows a representation of the considered measuring points. There are two different levels, one on the first floor of the gallery $(+6.6 \mathrm{~m}$.), and the other one on the start of the upper arches $(+12.2 \mathrm{~m})$. Accelerations were registered in $\mathrm{X}$ and $\mathrm{Y}$ directions with the aim of identifying the vibration modes in the lateral and longitudinal directions of the gallery. Eight accelerometers were used placing four of them as references (blue points in Fig. 9). The other four accelerometers were moved along the columns and the walls in the two different levels. In total, the resulting number of testing set-ups was fifteen.

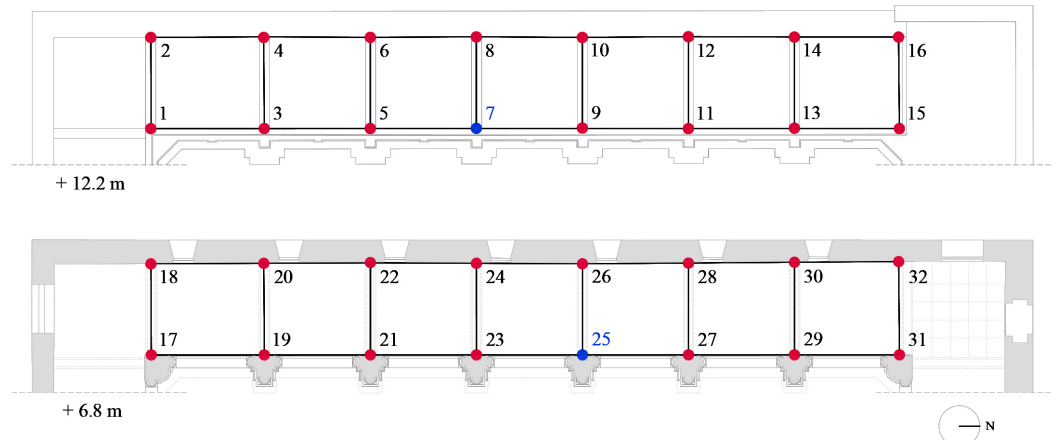

Fig. 9: Plan view of the accelerometer locations (reference accelerometers in blue).

The equipment used for these tests was composed of force balance accelerometers with a bandwidth ranging from 0.01 to $200 \mathrm{~Hz}$, a dynamic range of $140 \mathrm{~dB}$, a sensitivity of $10 \mathrm{~V} / \mathrm{g}$ and a mass of $0.35 \mathrm{~kg}$ (model ES-U2). These accelerometers were connected via eight $40 \mathrm{~m}$ long cables to a twelve-channel data acquisition system with a 24-bit ADC, provided with anti-alias filters (model GRANITE). The equipment is manufactured by the company KINEMETRICS. The parameters set for the dynamic tests were a sampling frequency of $100 \mathrm{~Hz}$ and approximately 15 minutes time duration for each test. These assumptions assure that frequencies from 1 to $50 \mathrm{~Hz}$ would be properly measured. Excitation was always associated with environmental ambient noise, and similar conditions of temperature and humidity were considered during the tests [32].

\subsection{OMA and model updating}

The data obtained in-situ were processed with the software ARTEMIS [33] using two different identification methods: Enhanced Frequency Domain Decomposition (EFDD) [34] and Stochastic Subspace Identification (SSI-UPC Merged) [35, 36] (Fig. 10). 

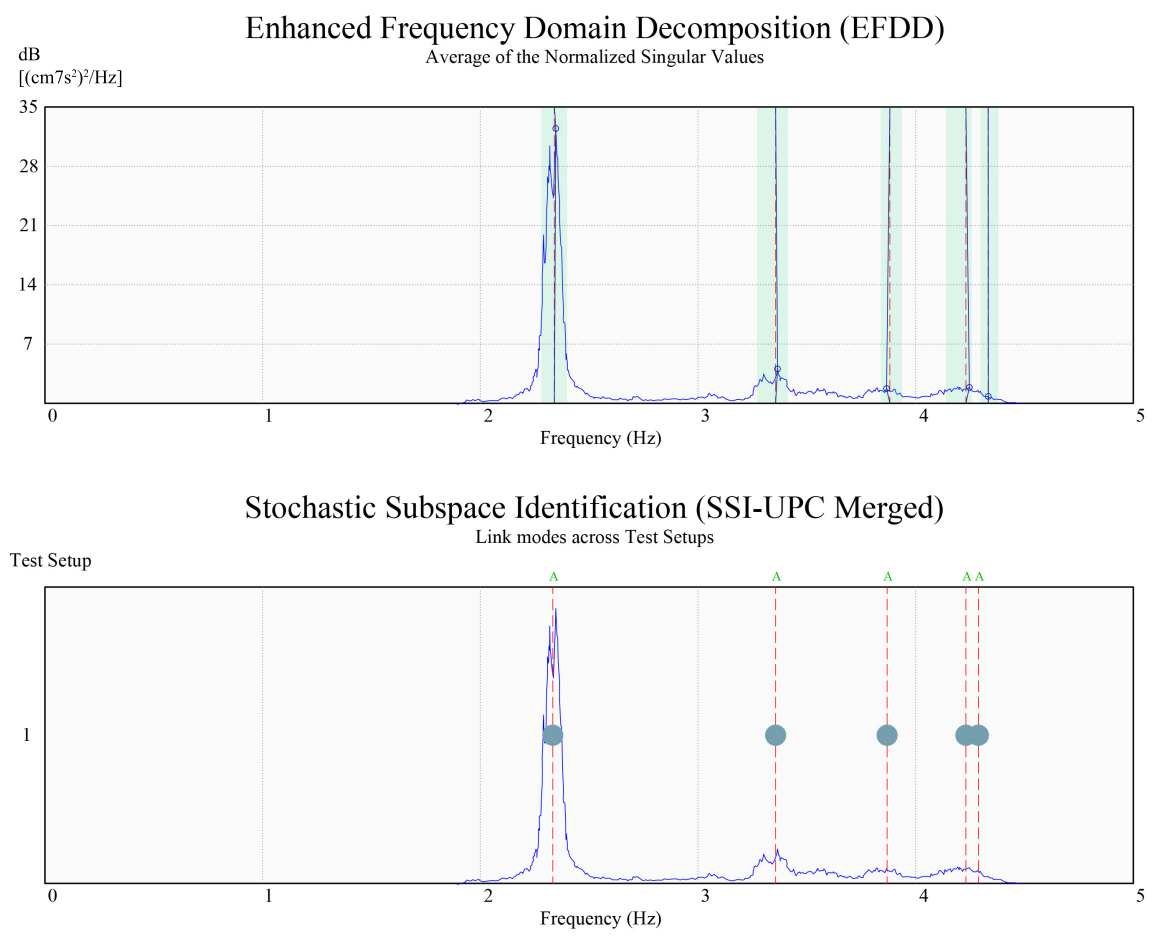

Fig. 10: Experimental identification by the EFDD and SSI-UPC-Merged methods.

Regarding the signal processing, a decimation factor of 5 and a spectral density resolution of 1024 was used, which leads to a frequency line spacing of $0.005 \mathrm{~Hz}$. In this way, the mode shapes and the modal frequencies of the gallery were obtained. The resulting modal parameters of the gallery are summarized in Table 2 .

Table 2: Experimental modal parameters: natural frequencies $(f)$, damping ratios $(\xi)$ and standard deviation (std).

\begin{tabular}{cccccccccc}
\hline \multirow{2}{*}{ Mode $\mathrm{N}^{\mathrm{o}}$} & \multicolumn{4}{c}{ EFDD } & & \multicolumn{4}{c}{ SSI } \\
\cline { 2 - 4 } \cline { 8 - 10 } & $f(\mathrm{~Hz})$ & $\operatorname{std}(f)$ & $\xi(\%)$ & $\operatorname{std}(\xi)$ & & $f(\mathrm{~Hz})$ & $\operatorname{std}(f)$ & $\xi(\%)$ & $\operatorname{std}(\xi)$ \\
\hline 1 & 2.330 & 0.023 & 0.864 & 0.119 & & 2.345 & 0.002 & 2.728 & 0.429 \\
2 & 3.359 & 0.021 & 1.292 & 0.468 & & 3.357 & 0.002 & 1.411 & 0.104 \\
3 & 3.857 & 0.049 & 0.582 & 0.307 & & 3.910 & 0.003 & 2.971 & 0.126 \\
4 & 4.311 & 0.056 & 1.565 & 0.799 & & 4.286 & 0.001 & 2.019 & 0.054 \\
5 & 4.561 & 0.042 & 1.348 & 0.473 & & 4.532 & 0.002 & 1.718 & 0.076 \\
\hline
\end{tabular}

As indicated in Table 2, the first five vibration modes of the gallery have been identified in the frequency range from 0 to $5 \mathrm{~Hz}$. Frequency values are obtained with a high degree of reliability, with differences between both methods always lower than $2 \%$. However, the values of the damping ratios present larger variability. This typical fact indicates that higher levels of excitation are usually necessary for the correct identification of the modal damping of such structures. Time domain methods have been proved to have better performance in the identification of damping ratios under operational conditions while the EFDD method, based on computation of the response spectrum 
data, usually requires longer records to obtain an acceptable error in spectrum estimation and, therefore, to extract modal parameters in a reliable way [37]. Finally, in relation to the mode shapes, with the exception of the first mode, which is a bending mode of the full gallery, all the modes are induced by the changing shape of the transverse arches of the first floor. The great complexity of these modes can be seen in Fig. 11.
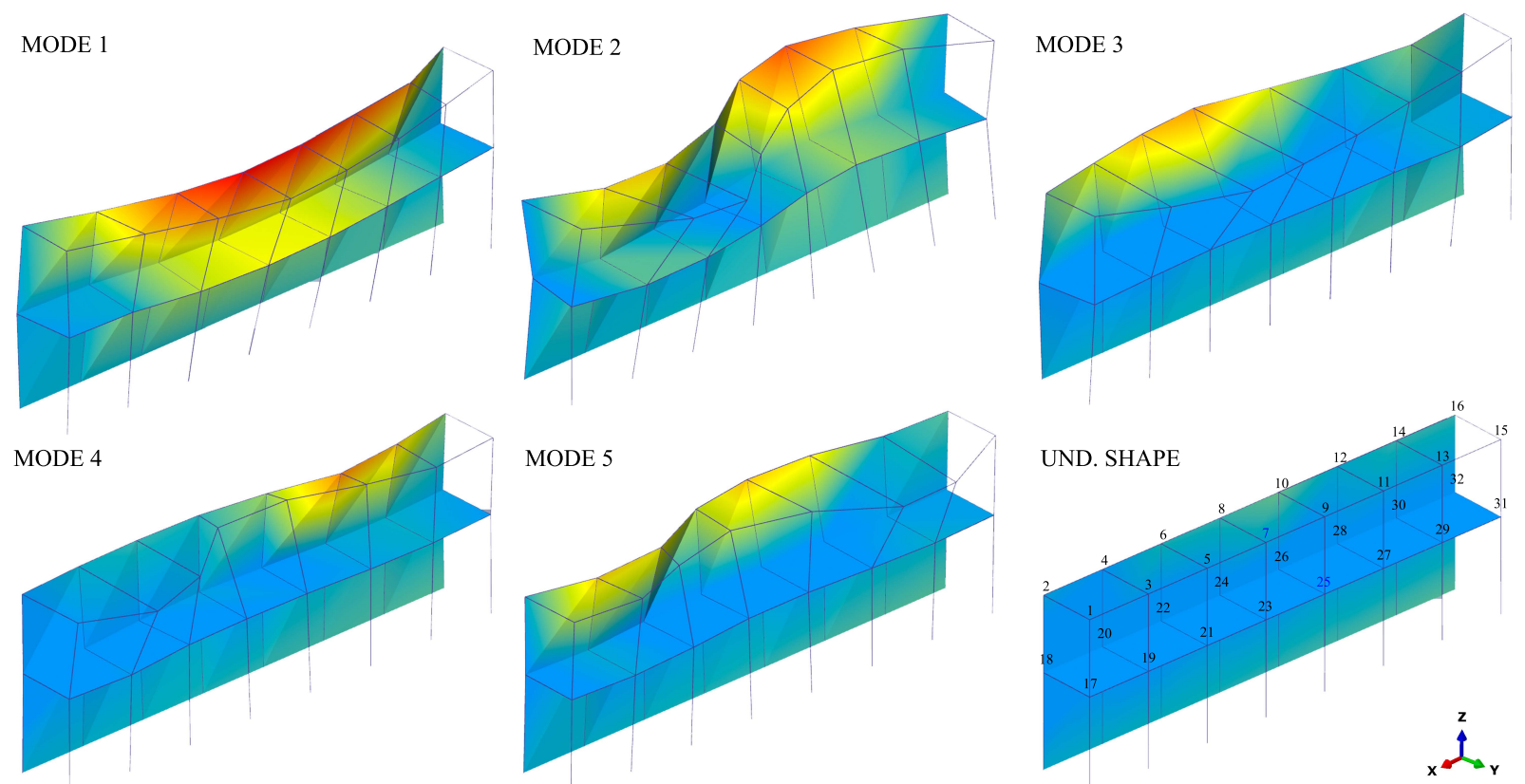

Fig. 11: First five experimental vibration modes and undeformed configuration of the experimental model.

Based on the experimental results, the preliminary numerical model is updated to simulate the current modal behaviour of the gallery and assist the evaluation of the OSP methods. The same procedure as in previous works by the authors was followed [38]. Hence, iterative methods are used to perform the FE model updating [39], introducing changes directly to some of the physical parameters that define the structure. As indicated above, the parameters to be updated are the Young's moduli of the stone and brick masonries due to the high degree of dispersion detected during the characterization tests. The selection of more parameters is not necessary, since their values have been reliably identified by laboratory tests.

Taking into account the good quality of the experimental data, the five identified modes are selected as target modes in the updating process. Only the values of the natural frequencies are selected due to the lower reliability of the identified mode shapes in comparison with the measured natural frequencies. This fact is due to the great complexity of the mode shapes (see Fig. 11). Nevertheless, the mode shapes are later validated using the Modal Assurance Criterion (MAC) [40]:

$$
\mathrm{MAC}_{j, k}=\frac{\left(\boldsymbol{\varphi}_{j}^{\mathrm{T}} \cdot \boldsymbol{\varphi}_{k}\right)^{2}}{\left(\boldsymbol{\varphi}_{j}^{\mathrm{T}} \cdot \boldsymbol{\varphi}_{j}\right) \cdot\left(\boldsymbol{\varphi}_{k}^{\mathrm{T}} \cdot \boldsymbol{\varphi}_{k}\right)}
$$

where $\varphi_{j}$ and $\varphi_{k}$ are two mode shapes to be compared and the superscript T designates transposition. 
Therefore, considering five identified natural frequencies, the four parameters are adjusted and the residuals are minimised during the model updating. The updating process has been performed via a genetic algorithm, as implemented in Matlab [41], according to an objective function defined by the relative differences between the experimental and the numerical modal parameters. This function is usually formulated as a least-squares problem as follows:

$$
l(\theta)=\frac{1}{2} \sum_{j=1}^{m} w_{j}\left[z_{N U M, j}(\theta)-z_{E X P, j}\right]^{2}=\frac{1}{2} \sum_{j=1}^{m} w_{j} r_{j}(\theta)^{2}
$$

where $z_{N U M, j}(\theta)$ are the values related to the physical parameters of the numerical model, $\theta\left(E_{c}\right.$ and $\rho_{c}$ ), while the variables $z_{E X P, j}$ are the corresponding data values obtained from the experimental campaign. The differences between these variables are set as residues, $r_{j}(\theta)$.

Fig. 12 illustrates the convergence of the updating process using a genetic algorithm. Taking as a reference the parameters of the preliminary model, a controlled variability range is established for the updating parameters. Thus, the estimation of unrealistic solutions is avoided (Table 3). In each iteration, a population of 1000 vectors is created that, by using the rules of the genetic algorithm, minimize the objective function in Eq. (2). The calibration process finishes when the difference between the mean value (blue points, Fig. 12) and the best value (black points, Fig. 12) of the population is less than $1 \times 10^{-3}$.

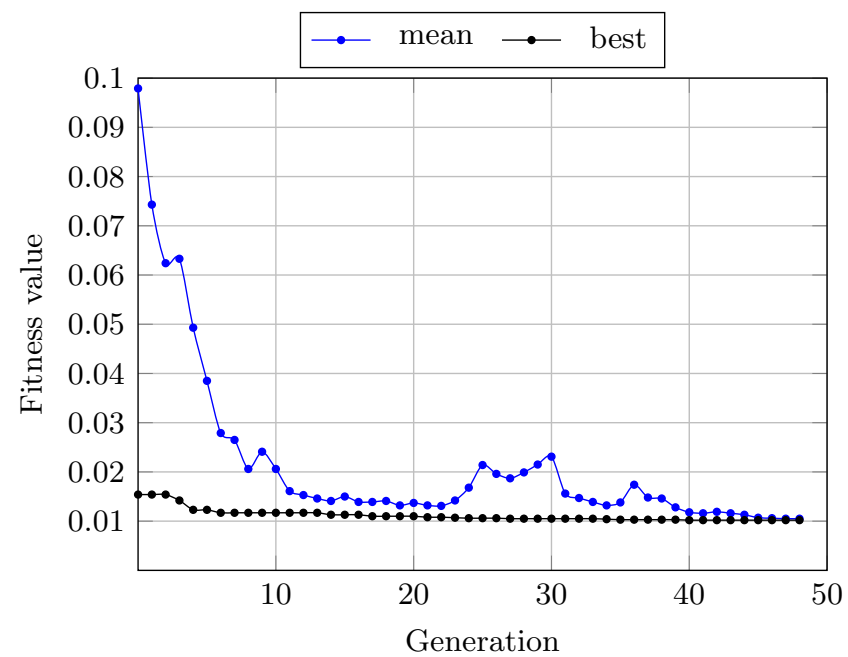

Fig. 12: Convergence plot of the genetic algorithm. Blue points: Mean values of the objective function of all the population of the corresponding generation. Black points: Best values result of an individual of the population.

Table 3 shows the considered lower and upper bounds for the updating parameters and their corresponding initial and updated values. The updated values differ by up to $20 \%$ with respect to those defined in the initial model. 
Table 3: Summary of updated parameters of the FE model computed by a genetic algorithm.

\begin{tabular}{ccccc}
\hline Parameter & Initial value & \multicolumn{2}{c}{ Range of variation } & \multirow{2}{*}{ Updated value } \\
\cline { 3 - 4 } & & Lower & Upper & \\
\hline$E_{b}(\mathrm{MPa})$ & 1300 & 900 & 1700 & 1000.51 \\
$\rho_{b}\left(\mathrm{~kg} / \mathrm{m}^{3}\right)$ & 1700 & 1300 & 2100 & 1805.72 \\
$E_{s}(\mathrm{MPa})$ & 1800 & 1200 & 2200 & 1520.36 \\
$\rho_{s}\left(\mathrm{~kg} / \mathrm{m}^{3}\right)$ & 2000 & 1600 & 2400 & 1954.86 \\
\hline
\end{tabular}

Table 4 summarizes the results following the updating process and confirms the high correspondence of the results between the calibrated model and those obtained from ambient vibration tests. It can be noted that the updated frequencies are close to the experimental ones, differing by less than $5 \%$ while exhibiting MAC values in a range from 0.82 (mode 3 ) to 0.98 (mode 1 ) for the five considered vibration modes.

Table 4: Comparison of numerical and experimental modal parameters, the latter identified by the SSI algorithm.

\begin{tabular}{cccccc}
\hline Mode & $f_{\text {exp }}(\mathrm{Hz})$ & $f_{\text {num }}(\mathrm{Hz})$ & MAC $_{\text {exp-num }}$ & $f_{\text {upd }}(\mathrm{Hz})$ & MAC $_{\text {exp-upd }}$ \\
\hline 1 & 2.345 & $2.534(8.05 \%)$ & 0.979 & $2.344(0.04 \%)$ & 0.980 \\
2 & 3.357 & $3.869(15.25 \%)$ & 0.915 & $3.510(4.77 \%)$ & 0.933 \\
3 & 3.910 & $4.126(5.52 \%)$ & 0.819 & $3.873(0.94 \%)$ & 0.825 \\
4 & 4.286 & $4.699(9.63 \%)$ & 0.872 & $4.380(2.19 \%)$ & 0.923 \\
5 & 4.532 & $4.947(9.15 \%)$ & 0.841 & $4.502(0.66 \%)$ & 0.862 \\
\hline
\end{tabular}

*The percentages in parenthesis correspond to the relative differences between frequencies.

\section{Sensor placement}

Vibration-based structural health monitoring is a suitable non-invasive technique for the longterm control of the condition of architectural heritage buildings. The placement of the sensors is a crucial design parameter of the monitoring system to efficiently identify the condition data. In this light, OSP techniques represent a very useful tool to design efficient monitoring set-ups, particularly in complex structures such as historical buildings.

After a concise theoretical background on OSP techniques, different OSP methods are applied to the present case study: the Monastery of San Jerónimo de Buenavista. The measuring points for the OSP problem are the same as for the ambient vibration tests (see Fig. 8). The chosen set-up has 32 possible locations for the sensors, with three different measuring directions at each point: i.e. the $x, y$ and $z$ directions. Therefore, a total of 96 possible sensor positions are considered in the OSP problem.

\subsection{Theoretical background}

Let us consider a monitoring system consisting of $n$ sensors for the dynamic identification of a structural system. Let us also assume that the system consists of $N$ degrees of freedom (DOFs) associated with the finite element mesh, and the numerical mode shapes $\varphi_{j}$ are extracted by 
performing modal analysis. Typically, only a limited number of DOFs and mode shapes of the FE model can be monitored due to accessibility or physical limitations (e.g. rotations or internal nodes). Therefore, considering $N_{d}$ candidate DOFs and $N_{m}$ target mode shapes, the OSP methods aim to identify the optimal positions of the $n$ sensors among the $N_{d}$ available DOFs. In this work, four different FEM-based OSP methodologies are considered, including two approaches based on the effective independence of the target mode shapes (EFI and EFIwm), and two others based on the concept of energy matrix rank optimization (KEMRO and SEMRO). In the following, the theoretical background of these techniques is concisely outlined.

The Effective Independence (EFI) method $[42,43]$ seeks the optimal location of sensors as those DOFs of the FE model that maximize the linear independence of the mode shapes. Based upon the modal analysis of the FE model, the target modal matrix $\varphi_{N_{d} \times N_{m}}$ can be computed, which contains the retained target modes as columns and the candidate DOFs as rows. Thereby, the Fisher Information Matrix, $\mathbf{F I M}_{N_{m} \times N_{m}}$, of the target modal matrix reads [44]:

$$
\mathbf{F I M}=\varphi^{\mathrm{T}} \boldsymbol{\varphi}
$$

which can be diagonalized by solving the eigenvalue/eigenvector problem:

$$
\left(\mathbf{F I M}-\lambda_{i} \mathbf{I}\right) \psi_{\boldsymbol{i}}=\mathbf{0}
$$

as $\mathbf{F I M}=\boldsymbol{\psi} \boldsymbol{\Lambda} \boldsymbol{\psi}^{-1}$, where $\boldsymbol{\Lambda}_{N_{m} \times N_{m}}$ is the diagonal matrix of the eigenvalues $\lambda_{i}$ of the FIM, and $\boldsymbol{\psi}_{N_{m} \times N_{m}}$ contains the corresponding eigenvectors $\boldsymbol{\psi}_{\boldsymbol{i}}$ by columns. Given that the FIM is symmetric and positive definite, the eigenvectors are orthogonal (i.e. $\boldsymbol{\psi}^{-1}=\boldsymbol{\psi}^{\mathrm{T}}$ ) and form an orthogonal basis in an $N_{m}$-dimensional space. Therefore, the product $\varphi \psi$ yields an $N_{d} \times N_{m}$ matrix that represents the projection of the mode shapes $\varphi_{i}$ onto the $N_{m}$-dimensional space spanned by the vectors $\boldsymbol{\psi}_{i}$. Squaring each element in the $\varphi \psi$ matrix as $\mathbf{A}=\boldsymbol{\varphi} \psi \otimes \varphi \psi$, with $\otimes$ denoting term-by-term matrix multiplication, the elements $A_{i j}$ of the resulting matrix represents the contribution of each $i$-th DOF to each $j$-th mode shape. If weighted by the inverse of the eigenvalue matrix of the FIM as $\overline{\mathrm{A}}=\boldsymbol{\varphi} \boldsymbol{\psi} \otimes \boldsymbol{\varphi} \boldsymbol{\psi} \Lambda^{-1}$, each element in this matrix has equal importance. The summation by columns of the $\overline{\mathbf{A}}$ matrix leads to a $N_{d} \times 1$ vector $\mathbf{F}$ whose components $F_{i}=\sum_{j=1}^{N_{m}} \overline{\mathbf{A}}_{i, j}$ represent the contribution of every $i$-th DOF to all the retained modes of interest. Alternatively, the orthogonal projection matrix $\mathbf{E}$ can be defined as [42]:

$$
\mathbf{E}=\varphi \psi \mathbf{\Lambda}^{-1}(\varphi \psi)^{\mathrm{T}}=\varphi \mathbf{F I M}^{-1} \varphi^{\mathrm{T}}
$$

The elements on the diagonal of the $\mathbf{E}$ projection matrix equals the components of the $\mathbf{F}$ vector and, therefore, represent the relative contribution of the candidate DOFs to the target mode shapes. It can be proved that the $\mathbf{E}$ matrix is idempotent [42], that is $\mathbf{E}^{2}=\mathbf{E}$. A well-known property of idempotent matrices is that their trace is equal to their rank. Therefore, the optimal placement of sensors can be related to the DOFs where the diagonal terms of $\mathbf{E}$ are maximum or, in other words, the DOFs with maximum contribution to the linear independence of the target mode shapes. In this light, the EFI method is defined in an iterative way by eliminating those candidate DOFs whose contribution to the rank of the projection matrix is minimal, and the procedure finishes when the number of remaining DOFs reaches the desired number of sensors.

An alternative OSP method considering the mass weighting of the effective independence of the target mode shapes is also considered, usually referred to as the EFIwm method. In this case, the Fisher information matrix FIM takes the form: 


$$
\mathbf{F I M}=\varphi^{\mathrm{T}} \mathbf{M} \boldsymbol{\varphi}
$$

and, consequently, the projection matrix $\mathbf{E}$ reads [45]:

$$
\mathbf{E}=\mathbf{M}^{1 / 2} \boldsymbol{\varphi} \mathbf{F I M}^{-1} \boldsymbol{\varphi}^{\mathrm{T}}\left(\mathbf{M}^{1 / 2}\right)^{\mathrm{T}}
$$

where $\mathbf{M}$ stands for the numerical mass matrix extracted from the FE model. Given that the mass matrix is diagonalizable, the square roots $\mathbf{M}^{1 / 2}$ in Eq. (7) can be readily obtained by eigendecomposition. In addition, in order to eliminate the DOFs with minimum contribution to the rank of $\mathbf{E}$, Guyan reduction must be implemented at each iteration to reduce the mass matrix $\mathbf{M}$ to the remaining candidate DOFs.

On the other hand, Energy Matrix Rank Optimization (EMRO) techniques are based upon the deployment of sensors at locations that maximize the energy of the monitored system, typically the strain energy or the kinetic energy [44]. Following the modal analysis of the FE model of the system, the strain energy ( $\mathbf{S E})$ and kinetic energy (KE) matrices can be written as [46, 47]:

$$
\mathrm{SE}=\varphi^{\mathrm{T}} \mathbf{K} \varphi, \quad \mathrm{KE}=\varphi^{\mathrm{T}} \mathrm{M} \varphi
$$

where $\mathbf{K}$ is the numerical stiffness matrix computed by the FEM. In this regard, the FIM matrices can be assembled by using the Cholesky decomposition of the stiffness and mass matrices as follows:

$$
\mathbf{K}=\mathbf{C}^{\mathrm{T}} \mathbf{C}, \quad \mathbf{M}=\mathbf{D}^{\mathrm{T}} \mathbf{D}
$$

with $\mathbf{C}$ and $\mathbf{D}$ being upper triangular matrices. Accordingly, the FIM matrices take the forms:

$$
\mathbf{F I M}_{S E}=(\mathbf{C} \varphi)^{\mathrm{T}} \mathbf{C} \boldsymbol{\varphi}, \quad \mathbf{F I M}_{K E}=(\mathbf{D} \varphi)^{\mathrm{T}} \mathbf{D} \boldsymbol{\varphi}
$$

and the projection matrices:

$$
\mathbf{E}_{S E}=\mathbf{C} \varphi \mathbf{F} \mathbf{I M}_{S E}^{-1}(\mathbf{C} \boldsymbol{\varphi})^{\mathrm{T}}, \quad \mathbf{E}_{K E}=\mathbf{D} \varphi \mathbf{F I M}_{K E}^{-1}(\mathbf{D} \varphi)^{\mathrm{T}}
$$

where subscripts "SE" and "KE" relate the corresponding quantities to the strain and kinetic energies, respectively. Once the projection matrices are defined, the procedure for finding the optimal sensor localizations is identical to the previously introduced EFI algorithms. In a similar way to the EFIwm method, Guyan reduction is needed to reduce the stiffness and mass matrices at each iteration. The OSP algorithms based on the elimination of candidate DOFs with minimum contributions to the rank of $\mathbf{E}_{K E}$ and $\mathbf{E}_{S E}$ matrices are referred to as the KEMRO and SEMRO methods, respectively.

\subsection{Identification of the optimal number of sensors}

The application of the above OSP techniques to the case study is now addressed. First of all, the required number of sensors is determined. Different scenarios were explored by varying the number of accelerometers of the monitoring system from 2 to 16 . The EFI method is employed to find the optimal configuration for each case. In order to analyse the goodness of the OSP solution, the data of the ambient vibration tests are used. The difference between the modal properties obtained via OMA using the complete test data (reference) and those obtained only by using 
the data from sensors placed at the optimal configuration is analysed. In particular, the error is computed as:

$$
\text { total error }(\%)=\left[\sum_{i=1}^{N_{m}} R_{i}^{2}\right]^{1 / 2}
$$

where $N_{m}$ is the total number of considered modes and $R_{i}$ is the relative error between the reference natural frequency of mode $i ; f_{r e f, i}$, and the frequency identified using only data from sensors in the OSP solution; $f_{o s p, i}$. That is:

$$
R_{i}(\%)=100 \cdot\left[\frac{f_{r e f, i}-f_{o s p, i}}{f_{o s p, i}}\right]
$$

In Fig. 13, the total error is shown for several cases considering different numbers of sensors in the OSP problem. Note that the error is stabilized for a number of sensors greater than 8 . Given these results, it can be considered that 8 is the optimal number of accelerometers for the case of the Monastery of San Jerónimo, since a reasonable increase in the number of sensors does not significantly reduce the error in the identification of the natural frequencies of the structure.

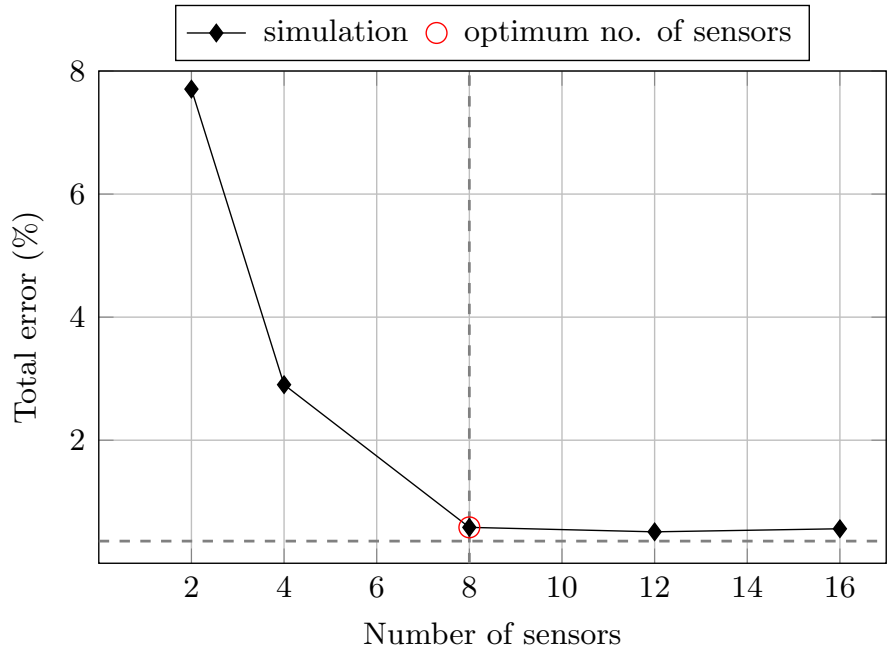

Fig. 13: Total error function for several scenarios with different numbers of sensors.

\subsection{Evaluation of the performance of OSP methods}

Once the required number of sensors for the long-term monitoring system has been determined, the OSP problem is performed to obtain the best positions for these 8 sensors. Four different OSP methods are evaluated, namely EFI, EFIwm, KEMRO and SEMRO.

In Fig. 14, the relative error in the identification of natural frequencies and the total error are shown for each method. For the EFI and EFIwm methods, the relative error is very low in all cases (below $0.5 \%$ ). In the case of the EMRO methods, the error is considerably greater. Especially for the KEMRO method, for which the error in the identification of the third natural frequency amounts to almost $6 \%$. As a partial conclusion, it can be said that the EFI methods allow the identification of the natural frequencies of the structure with a lower error. 


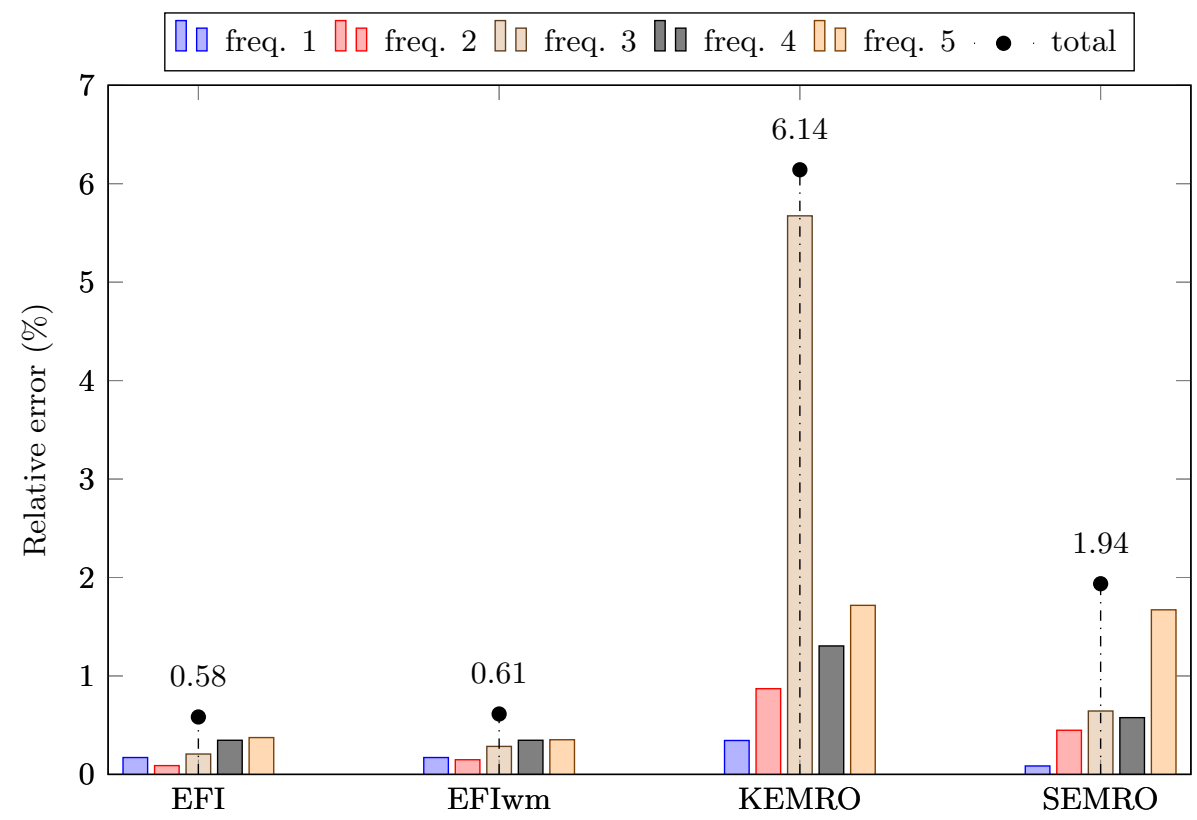

Fig. 14: Relative error function for different OSP methods.

Regarding the solution, the optimal locations of the 8 considered sensors obtained with the different OSP methods are shown in Fig. 15. In this bar plot, a chosen placement for a particular OSP method is represented by a bar of height 0.25 . In this way, a sensor location selected by all the methods is plotted with a value of 1 on the ordinate axis. There are three common positions that are selected by all the methods: points 5, 7 and 11, all in the $y$ direction. Another three points are selected by three of the four methods: points 6,10 and 12, all also in the $y$ direction. There is only one other common point chosen by both EFI methods: point 11 in the $x$ direction. The eighth optimal position could be chosen between any of the alternatives proposed by the different methods separately.

Although in a particular case the optimal locations would finally depend on the choice of one of the OSP algorithms, this analysis is useful for comparing the consistency of the different methods. As a general conclusion, the solution of the EFI, EFIwm and SEMRO methods are largely similar, while that of the KEMRO method is considerably different. 


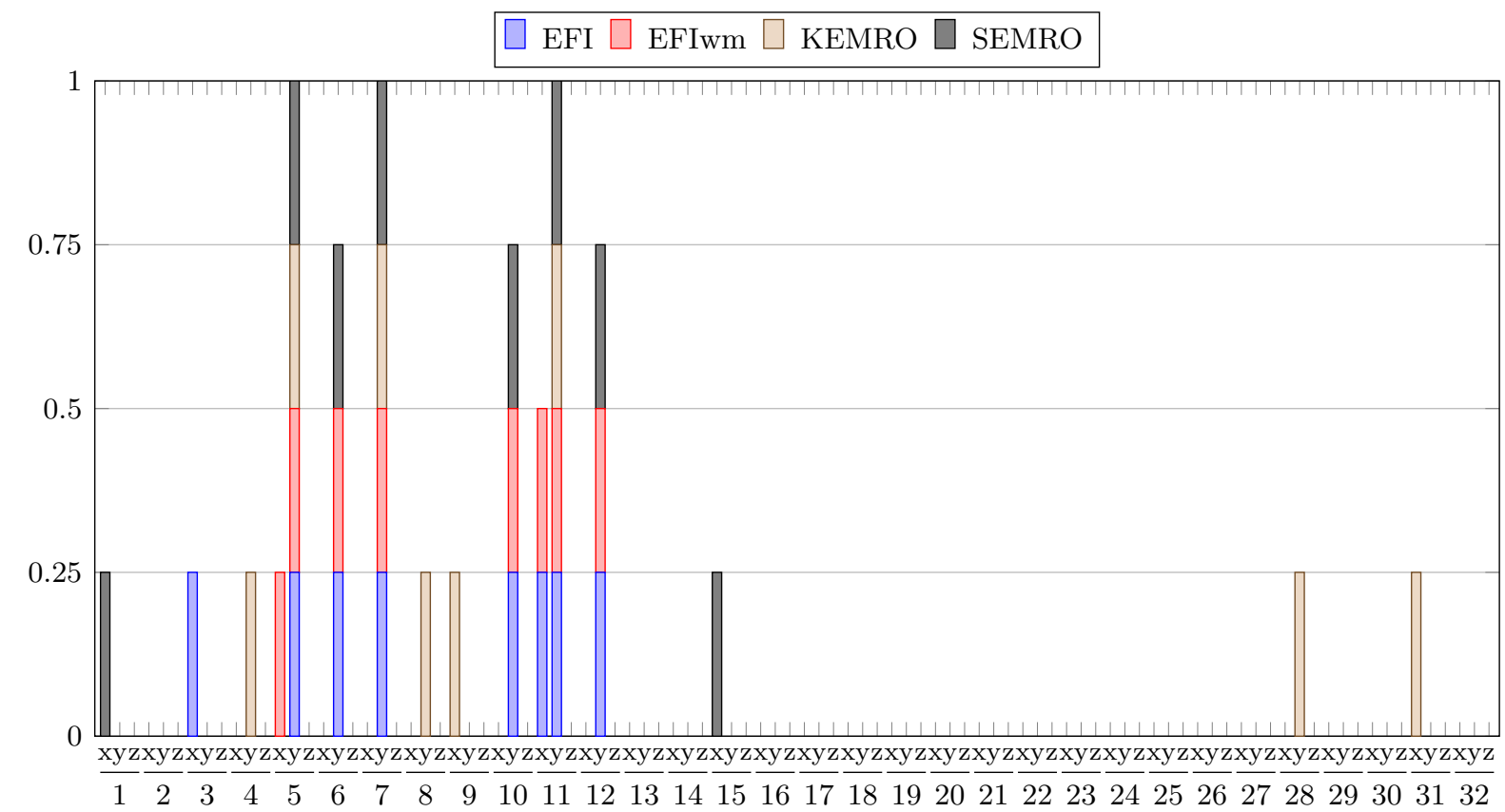

Fig. 15: Normalized sensor selection for different OSP methods considering deterministic material parameters.

\section{An optimal sensor placement strategy considering material parameter uncertainty}

This section assesses the robustness of the previously introduced OSP techniques considering material parameter uncertainties. In particular, the elastic moduli of the brick and the stone masonries are assumed to be stochastic with Gaussian distributions with mean values of $E_{b}=1000.51 \mathrm{MPa}$ and $E_{s}=1520.36 \mathrm{MPa}$, respectively, and standard deviations of $20 \%$ with respect to their mean values. The optimal selection of sensors is evaluated within a stochastic framework by applying Monte Carlo Simulations (MCS) as sketched in Fig. 16. Monte Carlo methods constitute a highly popular and widely used solution for the uncertainty propagation analysis of structures [48]. These techniques leverage the random description of certain model parameters, whereby the structural analysis can be performed by a deterministic simulation framework. Albeit these techniques usually require a large number of simulations and, as a consequence, considerable computational costs, the uncertainty analysis can be performed using the deterministic FE model. In this work, the MCS process first considers a random sampling of the elastic moduli of the brick and the stone masonries using MATLAB. Subsequently, the FE model of the monastery is built in the finite element modelling software ANSYS, and a modal analysis is performed to extract the mode shapes and natural frequencies. Afterwards, the previous information is retrieved in MATLAB, and the optimal positions of the sensors are obtained using the EFI, EFIwm, KEMO and SEMRO algorithms. Finally, this process is repeated $n s$ times until a statistically significant population of sensor locations is achieved. 


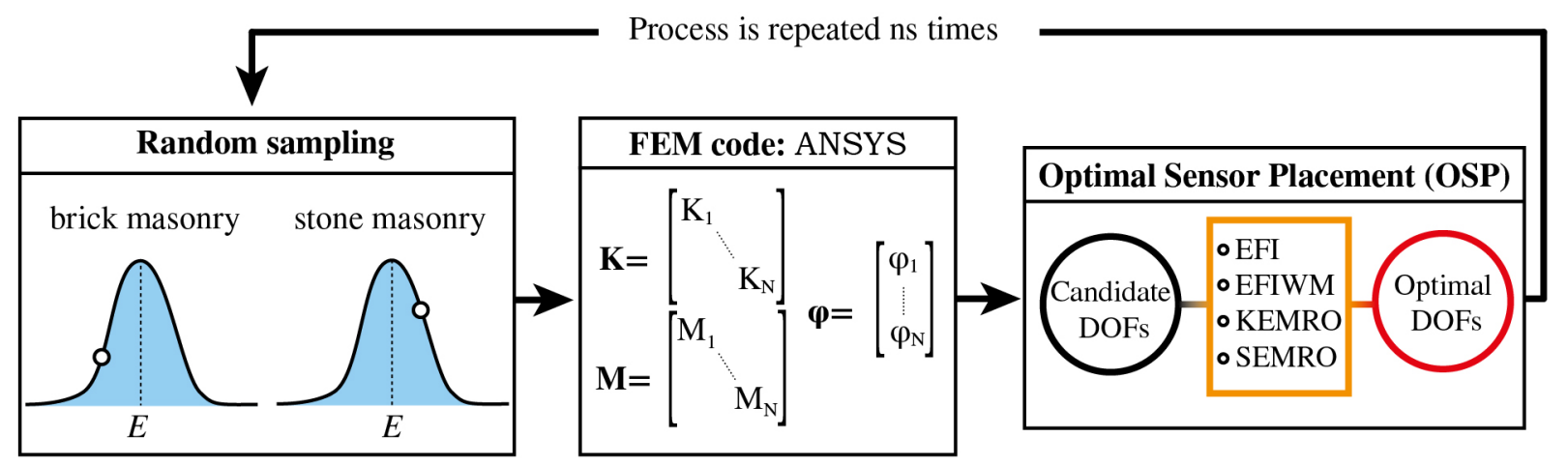

Fig. 16: The Monte Carlo simulation process.

\subsection{Determination of the optimal number of samples in the MCS}

An important parameter of the Monte Carlo approach is the number of samples $n s$ required to have a representative population of the simulated stochastic process. In this case study, several simulations with different number of samples from 2 to 516 were performed. The EFI method is employed in the OSP problem. In order to determine the optimum number of samples for the MCS, the dispersion percentage is calculated for each case. The dispersion is defined as the percentage of times during the MCS that the algorithm chooses a location which is finally not part of the solution, where the solution is represented by the eight sensors that are chosen most often. It can be computed as:

$$
\text { dispersion }(\%)=100 \cdot \frac{\sum_{i=1}^{n s} \bar{p}_{i}}{n s \cdot n}
$$

where $n s$ is the number of samples in the MCS, $n$ is the number of sensor of the monitoring system ( 8 in this case) and $\bar{p}_{i}$ represents the number of selected points for sample $i$ which are not present in the final solution.

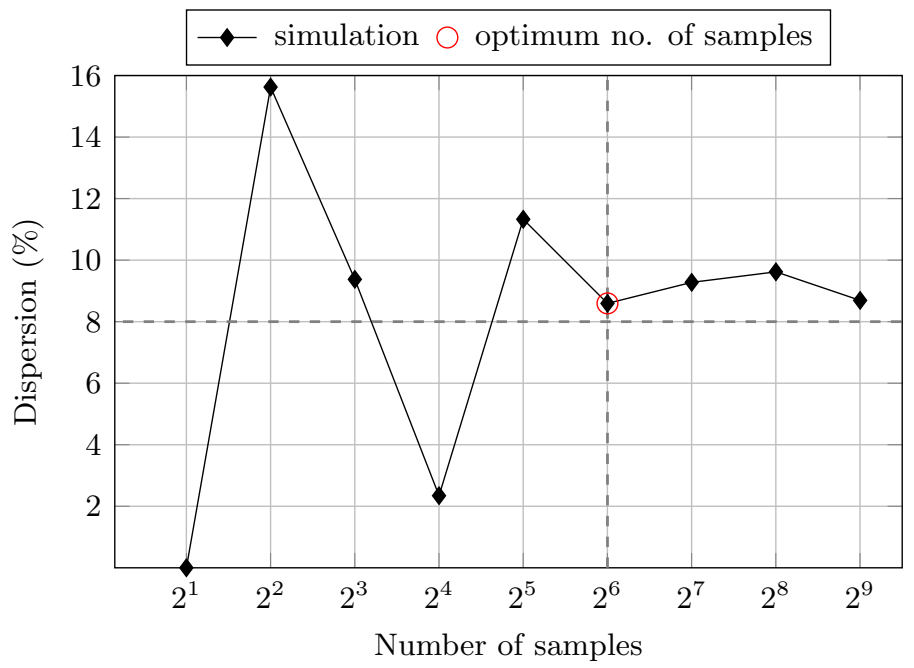

Fig. 17: Dispersion percentage for different number of samples in the MCS.

The dispersion percentage for MCS with different number of samples is presented in Fig. 17. For a low number of samples, the dispersion percentage experience strong fluctuations. However, 
the dispersion is stabilized for a number of samples greater than 64 . Consequently, 64 samples or more are considered in the following calculations.

\subsection{Effects of uncertainties on the OSP methods}

Once the minimum number of iterations for the MCS has been determined, the OSP problem is performed to evaluate the robustness of the different methods. For this purpose, first a Gaussian distribution of Young's moduli of brick and stone with standard deviations of $20 \%$ with respect to their mean values is considered. The dispersion percentage and the solution for each method is analysed.

In Fig. 18, the dispersion percentage computed with different numbers of samples of the MCS is shown for the four OSP methods. The EFI and KEMRO methods exhibit dispersion percentage about $10 \%$ and $22 \%$, respectively. The SEMRO method seems to be the most stable when considering uncertainty in the value of the model parameters, with less than $3 \%$ dispersion of the results.

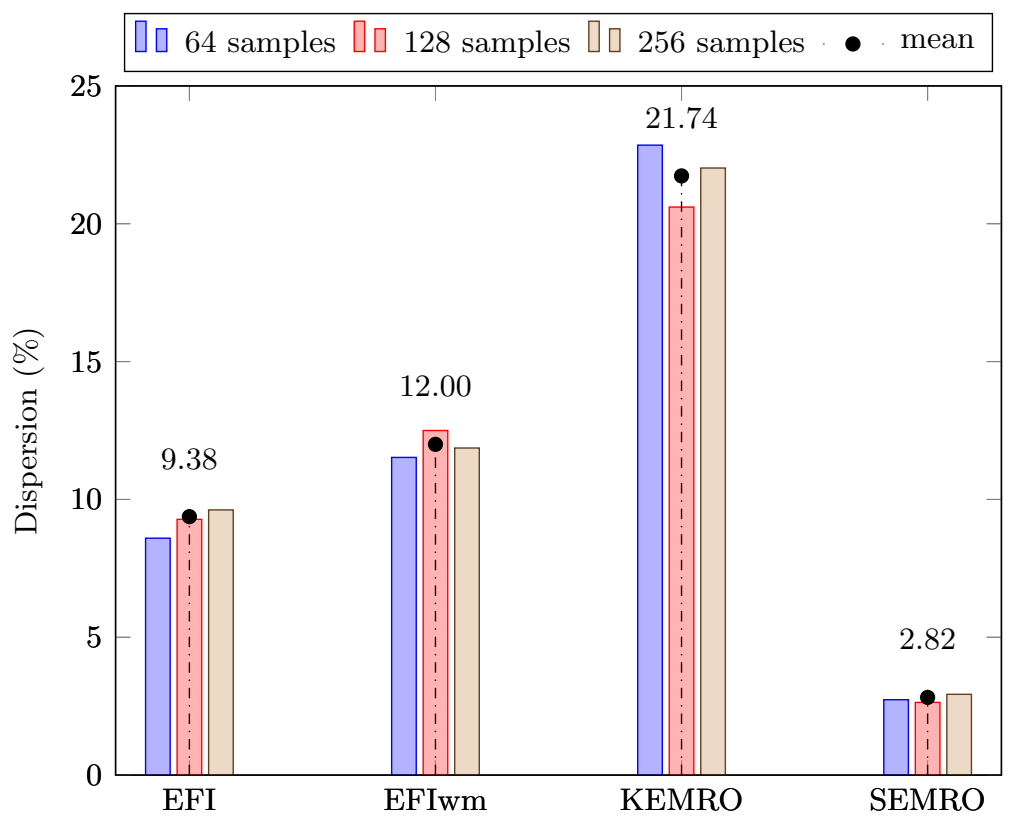

Fig. 18: Dispersion percentage for different OSP methods with $20 \%$ of parameter variability.

The solution considering a parametric variability of $20 \%$ is represented in Fig. 19 for different OSP methods. In this case, the probability of sensor selection is computed for each method and represented in the plot in a normalized form. There are two common positions selected by all the methods at all iterations: points 7 and 11, both in the $y$ direction. The point $5 y$ is also selected for the four methods with a combined probability greater than $95 \%$. As in the case with no parametric uncertainties, the points 6,10 and 12 in the $y$ direction are selected with a high probability for the EFI, EFIwm and SEMRO methods. Another interesting result is that the point $3 y$ is selected by the four methods but with a very low probability (below $25 \%$ ). In view of these results, the effect of considering a parameter variability of $20 \%$ does not strongly affect the solution of the OSP problem. 


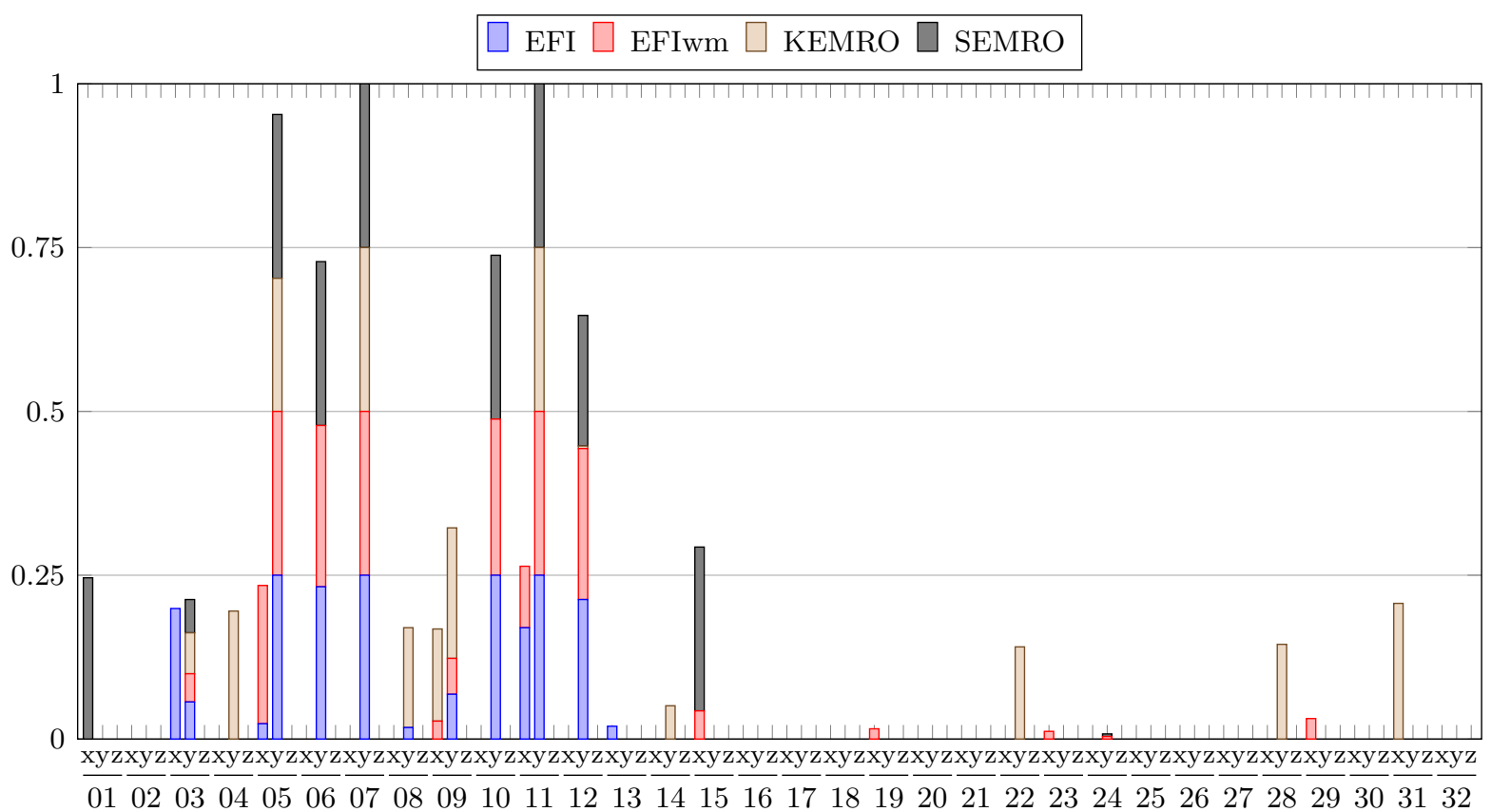

Fig. 19: Normalized probability of sensor selection for different OSP methods with $20 \%$ of parameter deviation.

Finally, different standard deviations for the brick and stone Young's moduli are considered. In particular, several percentages of variation between $2.5 \%$ and $40 \%$ are used to evaluate the robustness of the four considered OSP methods. The number of samples in the MCS is 64 . The results are presented in Fig. 20. There is a progressive increasing trend of dispersion as the variability of the parameters grows. The SEMRO method is again the one that seems to be more stable while the KEMRO generates a greater dispersion of the results in all cases. For the two EFI methods, the dispersion percentage is similar for the different standard deviation values of the parameters. 


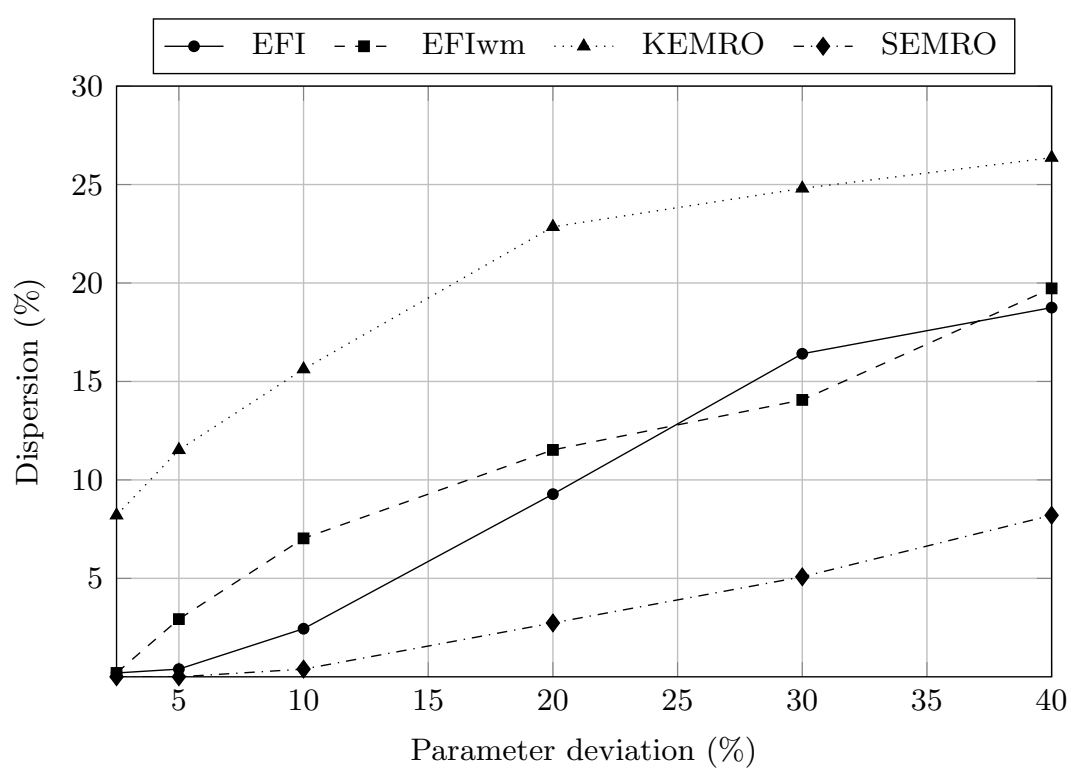

Fig. 20: Dispersion percentage versus parameter standard deviation for different OSP methods.

\section{Conclusions}

The paper has presented the application of the OSP methodology to historical masonry buildings such as the Monastery of San Jerónimo de Buenavista. The use of this technique becomes relevant in this kind of structures to reduce the number of sensors in a SHM monitoring system in order to be less-invasive. In this study, four different FEM-based OSP methodologies are analysed, including two approaches based on the concept of energy matrix rank optimization (KEMRO and SEMRO), and two others based on the effective independence of the target mode shapes (EFI and EFIwm). The modelling of historical buildings is usually subjected to a high level of uncertainty derived from numerous aspects such as material properties. Due to this, the effect of model parametric uncertainty on the results of the different OSP algorithms is also discussed in this work.

First, the optimal number of sensors for the monitoring system has been determined. The results demonstrate that, with only eight sensors, the natural frequencies of the structure could be identified with a maximum error less than $1 \%$ in comparison to the extensive monitoring with ninety-six measurement points. The suitability of the OSP techniques to find a convenient set-up for a cost-efficient continuous monitoring in complex structures is thus proven. Among the four OSP algorithms analysed, the EFI methods provide a solution that allows the identification of natural frequencies with less error, whereas the solution of the KEMRO method is the one that gives the greatest error in the modal identification.

Including parametric uncertainty in the OSP methodology involves performing Monte Carlo simulations with a sufficient number of samples to represent the stochastic process. The SEMRO method is the one that presents the lowest dispersion in its solution, for all the different scenarios analysed. A general conclusion for the case study presented is that the choice of the optimal sensor placements do not change drastically when considering a $20 \%$ variability in value of the parameters. 


\section{References}

[1] ICOMOS-ISCARSAH Committee, ICOMOS Charter-Principles for the analysis, conservation and structural restoration of architectural heritage, in: Proceedings of the ICOMOS 14th General Assembly and Scientific Symposium, Vol. 2731, 2003.

[2] C. Rainieri, A. Marra, G. Rainieri, D. Gargaro, M. Pepe, G. Fabbrocino, Integrated non-destructive assessment of relevant structural elements of an Italian heritage site: The Carthusian monastery of Trisulti, Journal of Physics: Conference Series 628 (1) (2015) 012018.

[3] S. Russo, Integrated assessment of monumental structures through ambient vibrations and ND tests: The case of Rialto Bridge, Journal of Cultural Heritage 19 (2016) 402-414.

[4] P. Van Overschee, B. De Moor, Subspace identification for linear systems: Theory-Implementation-Applications, Dordrecht, Holanda: Kluwer Academic Publishers, 1996.

[5] B. Peeters, System identification and damage detection in civil engineering, Ph.D. thesis, Katholieke Universiteit te Leuven (2000).

[6] C. Rainieri, G. Fabbrocino, Operational modal analysis of civil engineering structures-An introduction and guide for applications, Vol. 142, Springer, 2014.

[7] C. Rainieri, G. Fabbrocino, Development and validation of an automated operational modal analysis algorithm for vibration-based monitoring and tensile load estimation, Mechanical Systems and Signal Processing 60 (2015) $512-534$.

[8] L. F. Ramos, Damage identification on masonry structures based on vibration signatures, Ph.D. thesis, Universidade do Minho (2007).

[9] M. Diaferio, D. Foti, N. Giannoccaro, Identification of the modal properties of a building of the Greek heritage, Key Engineering Materials 628 (2014) 150-159.

[10] M. Diaferio, D. Foti, C. Gentile, N. Giannoccaro, A. Saisi, Dynamic testing of a historical slender building using accelerometers and radar, in: Proceedings of the 6th International Operational Modal Analysis Conference, 2015.

[11] C. Rainieri, G. Fabbrocino, G. Verderame, Non-destructive characterization and dynamic identification of a modern heritage building for serviceability seismic analyses, NDT and E International 60 (2013) 17-31.

[12] D. Foti, N. Giannoccaro, M. Diaferio, S. Ivorra, Structural identification and numerical models for slender historical structures, in: Handbook of Research on Seismic Assessment and Rehabilitation of Historic Structures, Vol. 2, IGI Global, 2015, Ch. 23, pp. 674-703.

[13] W. Torres, J. L. Almazán, C. Sandoval, R. Boroschek, Operational modal analysis and FE model updating of the Metropolitan Cathedral of Santiago, Chile, Engineering Structures 143 (2017) 169 - 188.

[14] C. Rainieri, A. Dey, G. Fabbrocino, F. S. de Magistris, Interpretation of the experimentally measured dynamic response of an embedded retaining wall by finite element models, Measurement 104 (2017) 316-325.

[15] C. Rainieri, G. Fabbrocino, E. Cosenza, Integrated seismic early warning and structural health monitoring of critical civil infrastructures in seismically prone areas, Structural Health Monitoring 10 (3) (2011) 291-308.

[16] C. R. Farrar, K. Worden, An introduction to structural health monitoring, Philosophical Transactions of the Royal Society A: Mathematical, Physical and Engineering Sciences 365 (1851) (2007) 303-315.

[17] K. Bernardeschi, C. Padovani, G. Pasquinelli, Numerical modelling of the structural behaviour of Buti's bell tower, Journal of Cultural Heritage 5 (4) (2004) 371-378.

[18] A. Saisi, C. Gentile, M. Guidobaldi, Post-earthquake continuous dynamic monitoring of the Gabbia Tower in Mantua, Italy, Construction and Building Materials 81 (2015) 101-112.

[19] F. Ubertini, G. Comanducci, N. Cavalagli, A. L. Pisello, A. L. Materazzi, F. Cotana, Environmental effects on natural frequencies of the San Pietro bell tower in Perugia, Italy, and their removal for structural performance assessment, Mechanical Systems and Signal Processing 82 (2017) 307-322.

[20] F. Lorenzoni, F. Casarin, C. Modena, M. Caldon, K. Islami, F. da Porto, Structural health monitoring of the Roman Arena of Verona, Italy, Journal of Civil Structural Health Monitoring 3 (4) (2013) 227-246.

[21] A. Elyamani, O. Caselles, P. Roca, J. Clapes, Dynamic investigation of a large historical cathedral, Structural Control and Health Monitoring 24 (3) (2016) e1885.

[22] A. D. Stefano, E. Matta, P. Clemente, Structural health monitoring of historical heritage in Italy: some relevant experiences, Journal of Civil Structural Health Monitoring 6 (1) (2016) 83-106.

[23] A. Afreen, A. Ahmed, K. Moin, State of art review: Structural health monitoring, retrofitting and rehabilitation of masonry structures, International Journal of Latest Engineering and Management Research 03 (02) (2018) $105-115$.

[24] L. F. Ramos, R. Aguilar, P. B. Lourenço, S. Moreira, Dynamic structural health monitoring of Saint Torcato church, Mechanical Systems and Signal Processing 35 (1) (2013) $1-15$. 
[25] D. Brigante, C. Rainieri, G. Fabbrocino, The role of the Modal Assurance Criterion in the interpretation and validation of models for seismic analysis of architectural complexes, Procedia Engineering 199 (2017) 3404-3409.

[26] E. Lenticchia, R. Ceravolo, C. Chiorino, Damage scenario-driven strategies for the seismic monitoring of XX century spatial structures with application to Pier Luigi Nervi's Turin Exhibition Centre, Engineering Structures 137 (2017) 256-267.

[27] E. Lenticchia, R. Ceravolo, P. Antonaci, Sensor placement strategies for the seismic monitoring of complex vaulted structures of the modern architectural heritage, Shock and Vibration (2018) art. no. 3739690.

[28] P. Pachón, R. Castro, E. García-Macías, V. Compán, E. Puertas, E. Torrojas bridge: Tailored experimental setup for SHM of a historical bridge with a reduced number of sensors, Engineering Structures 162 (2018) 11 21.

[29] F. Pozo, R. Gil, E. Méndez, Intervención arquitectónica puntual en Monasterio de San Jerónimo de Buenavista (Sevilla), Consejería de Urbanismo, 2003.

[30] J. García-Tapial, F. Sánchez, Proyecto de Rehabilitación del Monasterio de San Jerónimo de Buenavista para su adecuación a Centro Cívico, Servicio de Rehabilitación y Renovación Urbana, 2004.

[31] A. R. 14.0, ANSYS Users Manual (2011).

[32] L. Ramos, L. Marques, P. Lourenço, G. D. Roeck, A. Campos-Costa, J. Roque, Monitoring historical masonry structures with operational modal analysis: Two case studies, Mechanical Systems and Signal Processing 24 (5) (2010) 1291-1305.

[33] S. V. Solutions, Artemis modal 5.0. User's Guide (2015).

[34] T. Wang, O. Celik, F. Catbas, L. Zhang, A frequency and spatial domain decomposition method for operational strain modal analysis and its application, Engineering Structures 114 (2016) 104-112.

[35] B. Peeters, G. de Roeck, Reference-based stochastic subspace identificaiton for output only modal analysis, Mechanical Systems and Signal Processing 13 (6) (1999) 855-878.

[36] M. Döhler, P. Andersen, L. Mevel, Data merging for multi-setup operational modal analysis with data-driven ssi, in: Structural Dynamics, Vol. 3, Springer New York, 2011, pp. 443-452.

[37] C. Rainieri, G. Fabbrocino, E. Cosenza, Some remarks on experimental estimation of damping for seismic design of civil constructions, Shock and Vibration 17 (4-5) (2010) 383-395.

[38] P. Pachón, V. Compán, E. Rodríguez-Mayorga, A. Sáez, Control of structural intervention in the area of the Roman Theatre of Cadiz (Spain) by using non-destructive techniques, Construction and Building Materials 101 (2015) 572-583.

[39] A. Teughels, Inverse modelling of civil engineering structures based on operational modal data, Ph.D. thesis, University of Leuven (2003).

[40] R. J. Allemang, D. L. Brown, A correlation coefficient for modal vector analysis, in: Proceedings of the 1st International Modal Analysis Conference, 1982, pp. 110-116.

[41] MathWorks, MATLAB R2015a. User's Guide (2015).

[42] D. C. Kammer, Sensor placement for on-orbit modal identification and correlation of large space structures, Journal of Guidance, Control, and Dynamics 14 (2) (1991) 251-259.

[43] D. Kammer, L. Yao, Enhancement of on orbit modal identification of large space structures through sensor placement, Journal of Sound and Vibration 171 (1) (1994) 119-139.

[44] R. C. Triguero, S. Murugan, R. Gallego, M. I. Friswell, Robustness of optimal sensor placement under parametric uncertainty, Mechanical Systems and Signal Processing 41 (2013) 268-287.

[45] J. Lollock, T. Cole, The effect of mass weighting on the effective independence of mode shapes, in: Proceedings of the 46th AIAA/ASME/ASCE/AHS/ASC Structures, Structural Dynamics and Materials Conference, no. $1836,2005$.

[46] F. M. Hemez, C. Farhat, An energy based optimum sensor placement criterion and its application to structural damage detection, in: Proceedings of the 12th International Modal Analysis Conference, Vol. 2251, 1994.

[47] G. Heo, M. L. Wang, D. Satpathi, Optimal transducer placement for health monitoring of long span bridge, Soil dynamics and earthquake engineering 16 (7-8) (1997) 495-502.

[48] G. I. Schueller, Efficient Monte Carlo simulation procedures in structural uncertainty and reliability analysisrecent advances, Structural Engineering and Mechanics 32 (1) (2009) 1-20. 